\title{
Tyro3 is a podocyte protective factor in glomerular disease
}

\author{
Fang Zhong, ${ }^{1}$ Zhaohong Chen, ${ }^{2}$ Liwen Zhang, ${ }^{2}$ Yifan Xie, ${ }^{1,3}$ Viji Nair, ${ }^{4}$ Wenjun Ju, ${ }^{4}$ Matthias Kretzler, ${ }^{4}$ \\ Robert C. Nelson, ${ }^{5}$ Zhengzhe Li, ${ }^{1}$ Hongyu Chen, ${ }^{6}$ Yongjun Wang, ${ }^{6}$ Aihua Zhang, ${ }^{3}$ Kyung Lee, ${ }^{1}$ \\ Zhihong Liu, ${ }^{2}$ and John Cijiang He ${ }^{1,7}$ \\ 'Department of Medicine/Nephrology, Icahn School of Medicine at Mount Sinai, New York, New York, USA. ${ }^{2}$ National \\ Clinical Research Center of Kidney Diseases, Jinling Hospital, Nanjing University School of Medicine, Nanjing, China. \\ ${ }^{3}$ Department of Nephrology, Children's Hospital of Nanjing Medical University, Nanjing, China. ${ }^{4}$ Division of Nephrology, \\ University of Michigan, Ann Arbor, Michigan, USA. ${ }^{5}$ Chronic Kidney Disease Section, Phoenix Epidemiology and Clinical \\ Research Branch, National Institute of Diabetes and Digestive and Kidney Diseases (NIDDK), NIH, Bethesda, Maryland, \\ USA. ${ }^{6}$ Department of Nephrology, Hangzhou Hospital of Traditional Chinese Medicine, Zhejiang Chinese Medical \\ University, China. Renal Section, James J Peters Veterans Affair Medical Center, Bronx, New York, USA.
}

Our previous work demonstrated a protective role of protein $\mathrm{S}$ in early diabetic kidney disease (DKD). Protein $S$ exerts antiinflammatory and antiapoptotic effects through the activation of TYRO3, AXL, and MER (TAM) receptors. Among the 3 TAM receptors, we showed that the biological effects of protein $\mathrm{S}$ were mediated largely by TYRO3 in diabetic kidneys. Our data now show that TYRO3 mRNA expression is highly enriched in human glomeruli and that TYRO3 protein is expressed in podocytes. Interestingly, glomerular TYRO3 mRNA expression increased in mild DKD but was suppressed in progressive DKD, as well as in focal segmental glomerulosclerosis (FSCS). Functionally, morpholino-mediated knockdown of tyro3 altered glomerular filtration barrier development in zebrafish larvae, and genetic ablation of Tyro3 in murine models of DKD and Adriamycin-induced nephropathy (ADRN) worsened albuminuria and glomerular injury. Conversely, the induction of TYRO3 overexpression specifically in podocytes significantly attenuated albuminuria and kidney injury in mice with DKD, ADRN, and HIV-associated nephropathy (HIVAN). Mechanistically, TYR03 expression was suppressed by activation of TNF- $\alpha$ /NF- $\kappa B$ pathway, which may contribute to decreased TYRO3 expression in progressive DKD and FSCS, and TYRO3 signaling conferred antiapoptotic effects through the activation of AKT in podocytes. In conclusion, TYRO3 plays a critical role in maintaining normal podocyte function and may be a potential new drug target to treat glomerular diseases.

Authorship note: $\mathrm{FZ}, \mathrm{ZC}$, and $\mathrm{LZ}$ contributed equally to the work.

Conflict of interest: The authors have declared that no conflict of interest exists.

License: Copyright 2018, American Society for Clinical Investigation.

Submitted: July 16, 2018 Accepted: October 11, 2018 Published: November 15, 2018

Reference information: JCI Insight. 2018;3(22):e123482. https://doi.org/10.1172/jici. insight.123482.

\section{Introduction}

Podocyte loss occurs in both primary and secondary glomerular disease, leading to the progression of kidney disease $(1,2)$. Several studies demonstrated that podocyte number or density is strongly associated with the amount of proteinuria and decline of renal function (3). Therefore, large efforts have been devoted to develop specific treatments targeting podocyte injury (4). To develop such a therapy, it is critical to not only understand the mechanisms of podocyte injury, but also to identify molecules mediating the protective pathways against podocyte injury. A large body of evidence suggests that reduction in podocyte number in glomerular disease, such as focal segmental glomerulosclerosis (FSGS) and diabetic kidney disease (DKD), is caused by either detachment or apoptosis $(5,6)$. However, the protective mechanisms of podocyte injury in the early stage of glomerular diseases are much less understood. We recently performed a proteomic analysis of isolated glomeruli from diabetic rats with early DKD, and we identified protein S (PS) to be among the highly upregulated proteins in diabetic kidneys (7). Interestingly, expression of PS, which was significantly increased in the podocytes during the early stages of $\mathrm{DKD}$, was decreased at the late stages. We further showed that podocyte-specific $\mathrm{KO}$ of PS aggravated podocyte injury and progression of $\mathrm{DKD}$ in mice, whereas podocyte-specific overexpression of PS attenuated kidney injury, supporting a protective role of PS in DKD (7).

PS shares structural similarities with its homolog GAS6, which also plays an important role in the pathogenesis of kidney disease (8). Both PS and GAS6 bind to TYRO3, AXL, and MER (TAM) receptors 
that regulate various biological processes including cell survival, adhesion and migration, hemostasis, and inflammation (9). Although structurally similar, PS and GAS6 have disparate binding affinities to individual TAM receptors $(10,11)$, thereby leading to divergent functions (12). GAS6 binds with the strongest affinity to AXL, whereas PS does so to TYRO3 $(9,13)$. GAS6/AXL signaling induces mesangial cell proliferation and glomerular hypertrophy in early DKD through the activation of AKT/mTOR pathway (14, 15), promoting glomerular injury. In contrast, PS reduces cellular injury by negatively regulating immune and inflammatory responses via TYRO3 activation (16-18). Consistent with these observations, we recently showed that PS has antiinflammatory effects through activation of TYRO3 in cultured podocytes (7), suggesting that the renoprotective effects of PS in vivo are likely mediated by TYRO3.

The role of TYRO3 in podocyte function and glomerular disease, however, has not been previously explored. Herein, we demonstrate that TYRO3 expression is increased in the kidneys with glomerular disease in its early stages, but it is decreased in the kidneys with more advanced disease. Using cultured cells, zebrafish, and mouse models, our results clearly indicate a protective role of TYRO3 against podocyte injury.

\section{Results}

TYRO3 expression is altered in human and mouse kidneys with glomerulosclerosis. We first examined the expression pattern of TYRO3 in human kidneys using the Nephroseq database (http://www.nephroseq.org), which showed highly enriched TYRO3 mRNA expression in the glomeruli compared with other renal compartments (Supplemental Figure 1A; supplemental material available online with this article; https://doi. org/10.1172/jci.insight.123482DS1). It further showed that glomerular expression of TYRO3 was reduced in human diabetic kidneys compared with healthy controls (Supplemental Figure 1B) and that it positively correlated with the estimated glomerular filtration rate (eGFR) in diabetic patients (Supplemental Figure 1, $\mathrm{C}$ and D). TYRO3 expression in another set of biopsy samples from well-established DKD patients (European Renal cDNA Bank) similarly showed marked reduction of TYRO3 (Figure 1A). Interestingly, TYRO3 expression was increased in the kidney samples of type 2 diabetic Southwestern American Pima Indians with mild or early DKD (Figure 1B). Consistent with these observations in human kidneys, Tyro3 was increased in the glomeruli of diabetic mice with mild DKD, namely in $d b / d b$ in C57BLKS background and in streptozotocin-induced (STZ-induced) DBA/2J mice (Supplemental Figure 1E), but not in $d b / d b ; \mathrm{eNOS}^{-/-}$ mice with more progressive DKD (Supplemental Figure 1E). Collectively, these data indicate that TYRO3 expression is increased in the early stages of DKD with mild kidney injury but is reduced in more progressive DKD. Notably, this was also the expression pattern we found for PS, the ligand of TYRO3 (7).

In addition to diabetic kidneys, we also noted a reduction of glomerular TYRO3 expression in patients with FSGS (classic and collapsing) (Supplemental Figure 1F), as well as in a murine model of HIV-associated nephropathy with collapsing FSGS: Tg26 (19) (Supplemental Figure 1G). Therefore, we next examined the expression of TYRO3 in glomeruli of patients with nephrotic syndrome in collaboration with Nephrotic Syndrome Study Network (NEPTUNE). Of 128 adult patients with nephrotic syndrome, 26 reached composite endpoint of end-stage renal disease ESRD or $40 \%$ reduction of baseline eGFR during an average follow-up of 2.7 years. Baseline characteristics of the patients are shown in Supplemental Table 1. Cox proportional hazards regression analysis indicated that lower expression levels of TYRO3 mRNA in the glomeruli from the adult patients with primary glomerular disease were significantly associated with increased risk of progression to composite endpoints, with a hazard ratio (HR) of 0.54 ( $95 \% \mathrm{CI}, 0.38-0.84 ; P=0.006)$, independent of age, sex, and race; baseline eGFR and protein-to-creatinine ratio (20) (Figure 1C).

Immunofluorescence staining of TYRO3 protein in normal human kidney samples confirmed its predominant expression in the glomeruli and further demonstrated its colocalization with the podocyte marker synaptopodin, but not with the endothelial marker CD31 or the mesangial cell marker GATA3 (Figure 1D). Moreover, glomerular expression of both total TYRO3 and activated (phosphorylated) TYRO3 proteins were decreased in biopsy samples of DKD patients in comparison with nondiabetic controls (Figure 1, E and F). As clinically indicated, kidney biopsies are typically performed in patients with more progressive DKD — the significantly reduced TYRO3 expression is consistent with the above mRNA findings

Knockdown of tyro3 alters glomerular filtration barrier in zebrafish pronephros. We next examined the in vivo function of TYRO3 using the zebrafish pronephros as a model of glomerular maturation and the development of filtration barrier function (21). The zebrafish TYRO3 protein shares $\sim 48 \%$ homology with human TYRO3 protein (Supplemental Figure 2A). We confirmed the expression of tyro3 mRNA by reverse transcription PCR (RT-PCR) in isolated pronephric glomeruli of zebrafish $\mathrm{Tg}$ (pod:GFP) that expresses GFP 
A

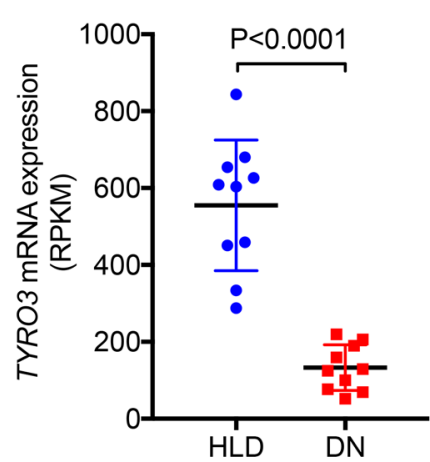

B

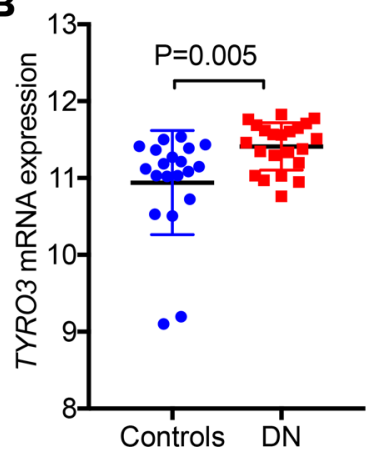

C

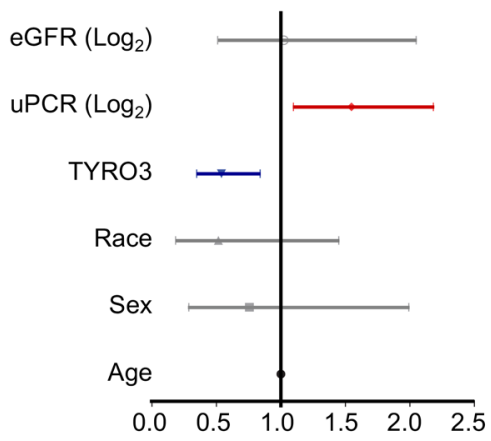

D

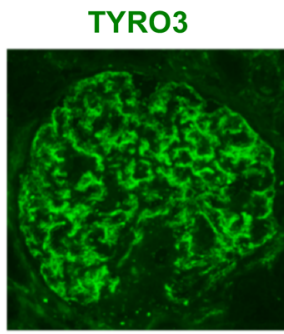

TYRO3

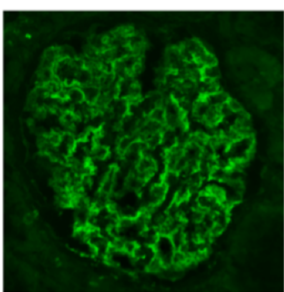

TYRO3

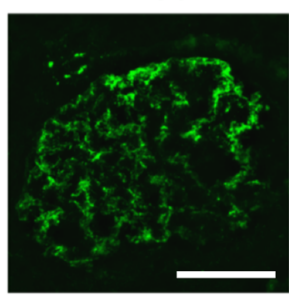

Synaptopodin

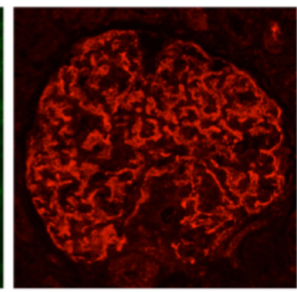

CD31

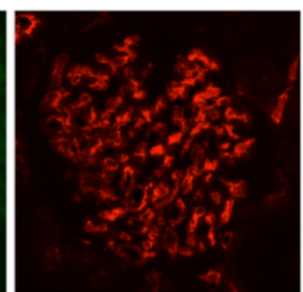

GATA3

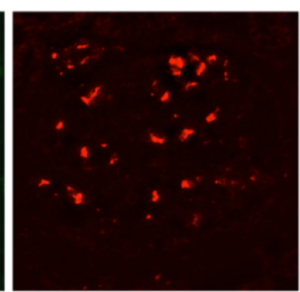

Merged

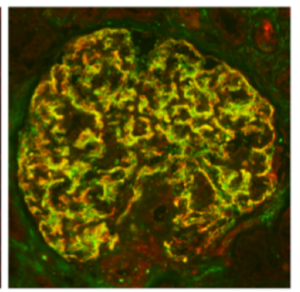

Merged

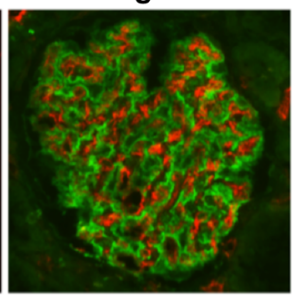

Merged

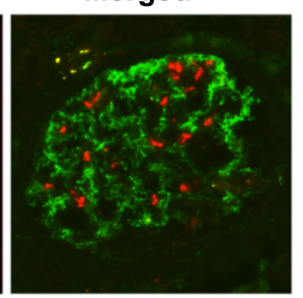

E

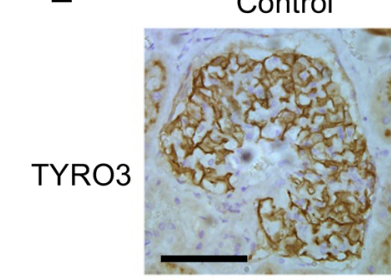

p-TYRO3

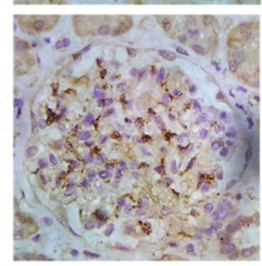

$\mathbf{F}$

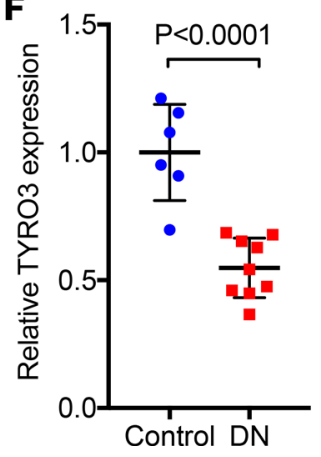

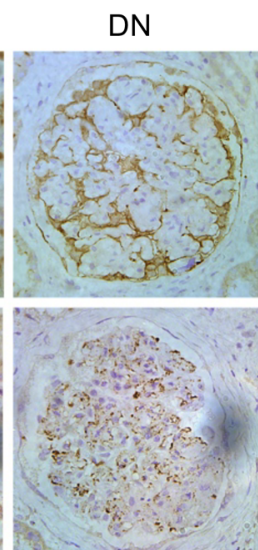

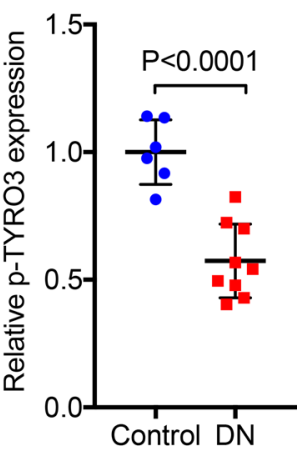

Figure 1. TYR03 expression in human diabetic kidneys. (A) TYRO3 mRNA expression in dissected glomeruli from healthy living donor (HLD) and progressive DN kidney biopsy samples (ERCB). $n=20$; statistical analysis was performed with 2-tailed, unpaired $t$ test. (B) TYRO3 mRNA expression in dissected glomeruli from HLD and DN kidney biopsy samples from Pima Indian diabetic cohorts. $n=42$; statistical analysis was performed with 2-tailed, unpaired $t$ test. (C) Association of glomerular TYRO3 mRNA with composite endpoint of ESRD or 40\% reduction of baseline eGFR. Results from multivariable Cox regression model are presented as hazard ratios with $95 \%$ confidence levels. Lower TYRO3 mRNA is markedly associated with outcome independent of age, sex, race, baseline eGFR and UPCR. eGFR, estimated glomerular filtration rate; UPCR, urinary protein to creatinine ratio. (D) Immunofluorescence staining of TYRO3 with podocyte marker synaptopodin, endothelial marker CD31, or mesangial marker GATA3 in normal human kidneys. (E) Immunohistological staining of TYRO3 and phospho-TYRO3 (p-TYRO3) in normal and DN human kidneys. (F) Semiquantitative analysis of TYRO3 and p-TYRO3 immunostaining. $n=6$ in control, $n=9$ in DN; statistical analysis was performed with 2-tailed, unpaired $t$ test.

transgene under nphs2 (podocin) promoter (Supplemental Figure 2B). We utilized morpholino-oligo (MO) to specifically block the mRNA splicing of tyro3 (Supplemental Figure 2, C and D). Real-time PCR and sequencing confirmed a reduction in tyro3 mRNA in tyro3 MO-injected zebrafish (tyro3-MO) in comparison with control MO-injected zebrafish (CTL-MO) (Supplemental Figure 2, E and F).

We observed that $\sim 55 \%$ of tyro3-MO developed pericardial and periorbital edema, the hallmark of glomerular failure, in comparison with CTL-MO at 72 hours after fertilization (hpf) (Figure 2, A and B). The integrity of the glomerulus filtration barrier (GFB) was measured by using an established pupillary 
A

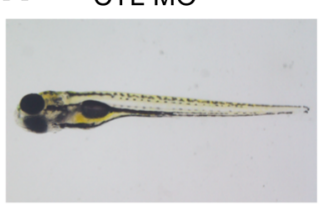

C

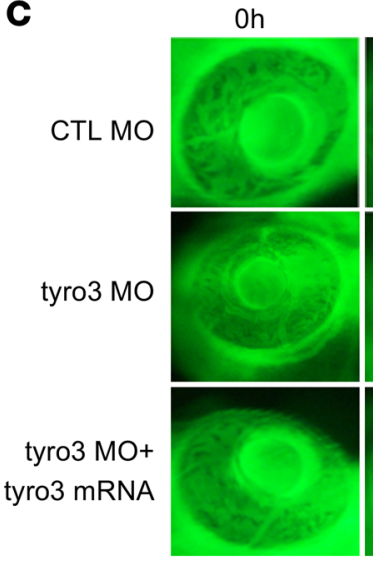

tyro3 MO

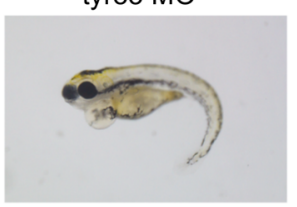

tyro3 MO+tyro3 mRNA

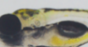

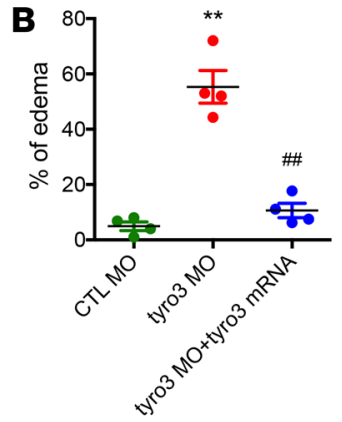

E

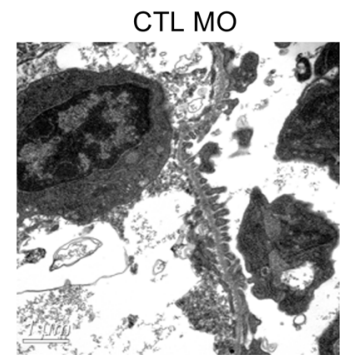

$24 \mathrm{~h}$

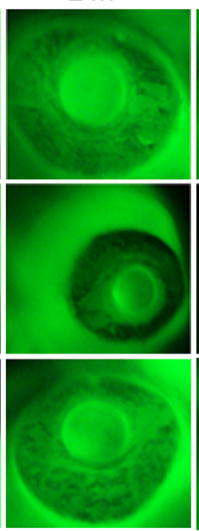

$36 \mathrm{~h}$

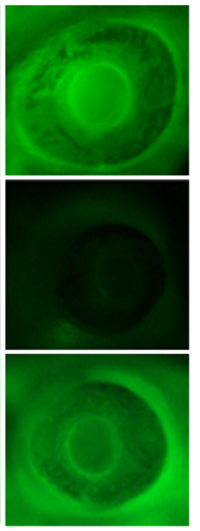

D

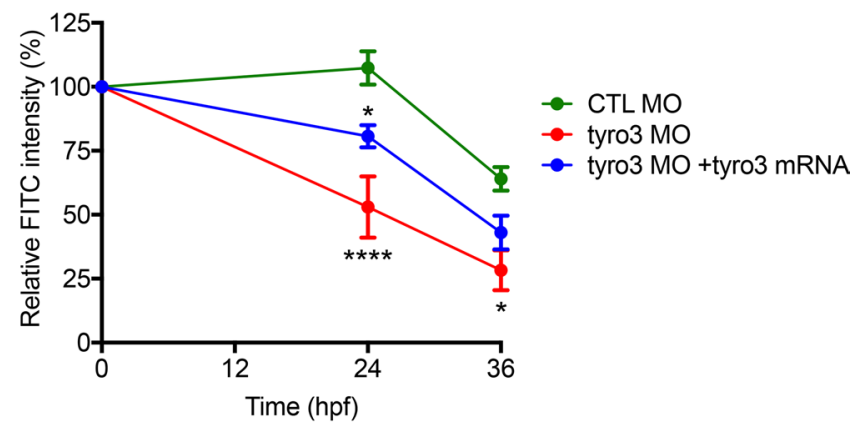

tyro3 $\mathrm{MO}$

tyro3 MO+tyro3 mRNA

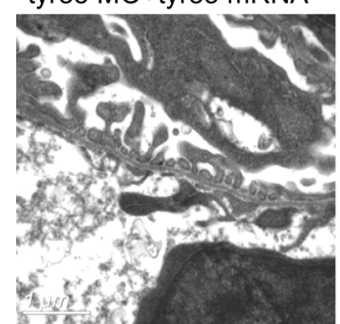

Figure 2. Knockdown of tyro3 in zebrafish larvae compromises the glomerular filtration barrier. (A) Representative images of zebrafish that were control morpholino treated (CTL MO), tyro3 morpholino treated (tyro3 $\mathrm{MO}$ ), or tyro3 $\mathrm{MO}$ rescued with tyro3 mRNA (tyro3 MO+tyro3mRNA) in zebrafish larvae at 72 hours after fertilization (hpf). (B) Quantification of pericardial and periorbital edema in MO-treated zebrafish larvae. Data are shown as mean \pm SEM. Statistical analysis was performed with 1-way ANOVA with Tukey's multiple comparison test; ${ }^{* *} P<0.01$ vs. CTL MO and \#\# $P<0.01$ vs. tyro3 MO. (C and D) Pupillary fluorescence intensity assay using FITC-dextran injection at 72 hours hpf. Quantification of FITC intensity relative to 0 houfs is shown in $\mathbf{D}$. Magnification, $20 \times$. Data are shown as mean \pm SEM. Statistical analysis was performed with 2-way ANOVA with Tukey's multiple comparison test; ${ }^{*} P<0.05$ and ${ }^{* * * *} P<0.0001$ vs. control. (E) Representative transmission electron microscopy images of pronephros. Red arrow highlights the area of substantial foot process effacement.

fluorescence assay. High-molecular weight FITC-dextran $(70 \mathrm{kDa})$ was injected via the cardinal vein at 72 $\mathrm{hpf}$, and the pupillary fluorescence intensity was monitored at 0,24 , and 36 hours after injection. In CTLMO, the injected FITC-dextran was largely retained even at 36 hours after injection, whereas in tyro3-MO, the fluorescence intensity diminished rapidly, indicating that the GFB is significantly compromised in tyro3-MO (Figure 2, C and D). Consistent with these findings, the ultrastructural analysis by electron microscopy (EM) revealed marked foot process effacement and disrupted slit diaphragms in tyro3-MO without obvious abnormalities in the endothelial layer or the GBM (Figure 2E). To verify whether the impaired GFB and podocyte injury were specifically induced by tyro3 knockdown (TYRO3 ${ }^{\mathrm{KD}}$ ), we injected tyro3 mRNA together with tyro3 $\mathrm{MO}$ (tyro3-MO + tyro3 mRNA). The restoration of tyro3 mRNA levels dramatically improved edema (Figure 2, A and B), GFB function (Figure 2, C and D), and foot process effacement induced by TYRO3 ${ }^{\mathrm{KD}}$ (Figure 2E). These data indicate the necessity of TYRO3 in maintaining glomerular filtration barrier and podocyte foot processes in development of zebrafish pronephros.

Genetic loss of Tyro3 aggravates diabetic nephropathy in mice. To explore the role of TYRO3 in diabetic kidney injury, we examined the effects of global genetic loss of Tyro3 in the setting of experimental DKD. Tyro3-null mice in C57BL/6J background was obtained from The Jackson Laboratory, and their WT littermates were used as controls. The loss of TYRO3 in Tyro3-null mice was confirmed by a Western blot of 
kidney cortices (Supplemental Figure 3A). Diabetes was induced by multiple injections of low-dose STZ in 8-week-old Tyro3 WT and -null mice (STZ-WT and STZ-KO). Sodium citrate buffer-injected mice served as nondiabetic controls (WT and KO). All mice were euthanized after 20 weeks of diabetes induction. The levels of hyperglycemia or body weight loss following diabetes induction were unremarkable between STZ-WT and STZ-KO mice (Supplemental Figure 3, B and C). However, diabetes-induced kidney hypertrophy was more pronounced in KO-STZ than in WT-STZ mice (Supplemental Figure 3D). KO-STZ mice displayed worsened albuminuria and mesangial expansion as compared with WT-STZ mice (Figure 3, A-E). Under EM, more pronounced podocyte foot process effacement was observed in KO-STZ glomeruli compared with WT-STZ glomeruli (Figure 4, A and B). Quantitative PCR (qPCR) indicated a reduction in podocyte marker Wilm's tumor-1 (WT1) expression in KO-STZ in comparison with WT-STZ (Figure 4C). Consistently, podocyte number, as ascertained by $\mathrm{WT}^{+}$nucleus per glomerular cross section or glomerular tuft area, was decreased in KO-STZ in comparison with WT-STZ (Figure 4, D-E). Consistent with our previous report of PS in the diabetic kidney (7), we also observed a heightened expression of inflammatory markers in KO-STZ mice compared with WT-STZ mice (Supplemental Figure 3E). Together, these results indicate that the loss of TYRO3 aggravates diabetic injury and podocyte loss in DKD.

Genetic loss of Tyro3 aggravates Adriamycin-induced nephropathy. We next determined whether the loss of Tyro3 also aggravated glomerulosclerosis in the setting of Adriamycin-induced nephropathy (ADRN). Eight-week-old WT and KO mice were injected with Adriamycin $(18.8 \mathrm{mg} / \mathrm{kg})$ and sacrificed 28 days after injection. Loss of Tyro3 exacerbated albuminuria and glomerulosclerosis (Figure 5) and worsened podocyte foot process effacement and podocyte loss (Figure 6). Similar to the above findings in diabetic mice, these results indicate that the loss of Tyro3 aggravates kidney injury and podocyte loss in mice with ADRN.

Induction of TYRO3 expression in podocytes ameliorates diabetic nephropathy. Because TYRO3 expression was found predominantly in podocytes in the glomeruli and genetic loss of Tyro3 led to worsening of glomerular diseases, we next sought to determine whether the increased Tyro3 specifically in podocytes would mitigate podocyte and glomerular injury. Positive results would not only help determine the specific effects of TYRO3 in podocytes, but it would also indicate whether TYRO3 could be a potential therapeutic target of DKD and other glomerular diseases. Tetracycline-inducible podocyte-specific Tyro3 overexpression mice (NPHS2-rtTA;TRE-Tyro3; hereafter referred to as TYRO3 overexpression [Tyro3 ${ }^{\mathrm{OV}}$ ]) in FVB/N background were generated, as described previously $(22,23)$. Doxycycline-induction of TYRO3 expression was confirmed by Western blot and real-time PCR analysis (Supplemental Figure 4A). We first checked the effect of TYRO3 overexpression in the experimental model of DKD. Tyro3 ${ }^{\text {OV }}$ was crossed with OVE26 mice, a transgenic model of severe early-onset type 1 diabetes in the FVB/N background $(24,25)$. Since OVE26 mice usually develop pronounced albuminuria by the age of $8-10$ weeks, we induced the podocyte-specific TYRO3 overexpression starting at 8 weeks of age in OVE26;Tyro3 ${ }^{\text {ov }}$ with doxycycline-supplemented chow (DOX; $625 \mathrm{mg} / \mathrm{kg}$ ) for 4 weeks to maintain high expression of TYRO3 during the disease course (Supplemental Figure 4B). Control OVE26;Tyro3 ${ }^{\mathrm{OV}}$ without DOX supplementation, and WT and OVE26 mice with DOX treatment, were used as controls. Irrespective of DOX-induced TYRO3 expression, all OVE26 mice displayed a similar extent of hyperglycemia (Figure 7A). However, diabetes-induced kidney hypertrophy was observed in all OVE26 mouse groups, except in the OVE26;Tyro3 ${ }^{\text {OV }}+$ DOX mice (Figure 7B). Moreover, OVE26; Tyro3 ${ }^{\mathrm{OV}}+$ DOX mice showed marked attenuation in albuminuria and glomerulosclerosis (Figure 7, C-F). Consistent with the renoprotection by TYRO3 overexpression, podocyte loss was also diminished in OVE26; Tyro3 ${ }^{\mathrm{OV}}+$ DOX mice (Figure 8).

Induction of TYRO3 expression in podocytes ameliorates ADRN and HIV-associated nephropathy. We next explored whether the podocyte-specific Tyro3 overexpression would attenuate other glomerular diseases. To examine its role in ADRN, Tyro3 overexpression in podocytes was induced on the day the mice received the injection of adriamycin (ADR). While Tyro3 mRNA expression was decreased in ADR-injected WT mice in comparison with vehicle-injected WT controls, as anticipated, its levels were greater in TYRO3 ${ }^{\mathrm{OV}}$ mice in comparison with WT with or without ADR (Supplemental Figure 4C). Induction of Tyro3 overexpression in the ADRN mice markedly decreased the extent of albuminuria and glomerulosclerosis (Figure 9), as well as podocyte foot process effacement and loss (Supplemental Figure 5). The effects of TYRO3 overexpression were also examined in the setting of HIVAN in HIV-1 transgenic Tg26 mice. Tg26 mice usually develop proteinuria at the age of 4 weeks. Therefore, we induced Tyro3 overexpression in podocytes of Tg26 mice by feeding DOX starting at 4 weeks of age, which maintained high expression of Tyro3 to 8 weeks, when the mice were euthanized (Supplemental Figure 4D). The induction of Tyro3 overexpression in podocytes 
A

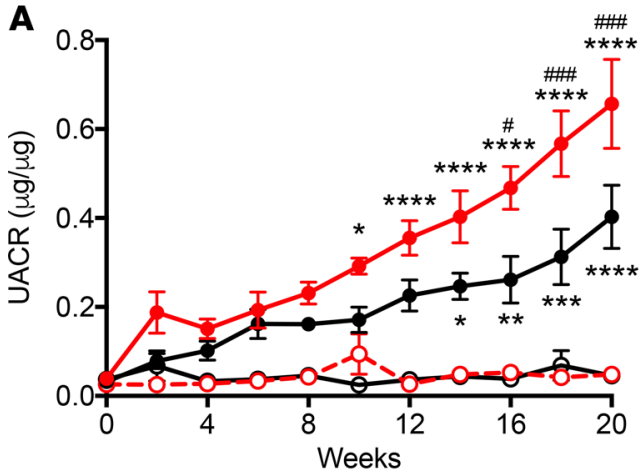

B

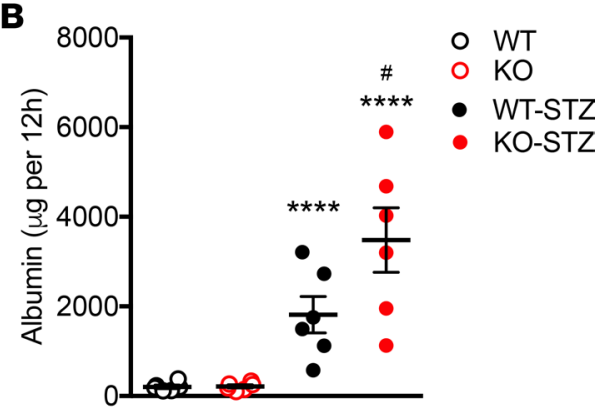

C

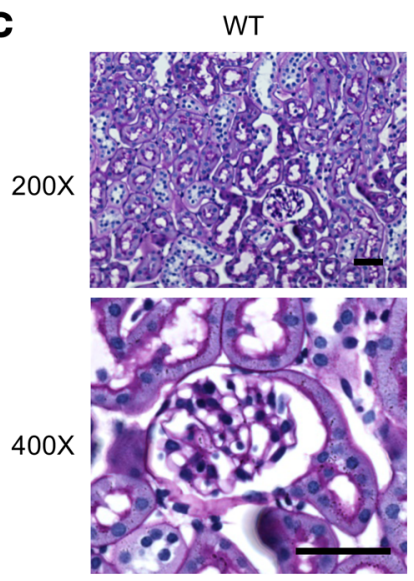

$\bullet W T$

- $\mathrm{KO}$

$\leadsto$ WT-STZ

$\rightarrow$ KO-STZ
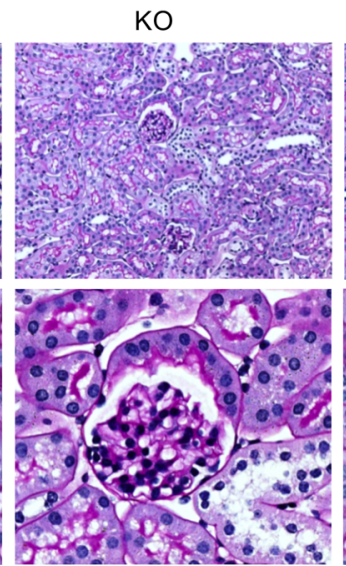

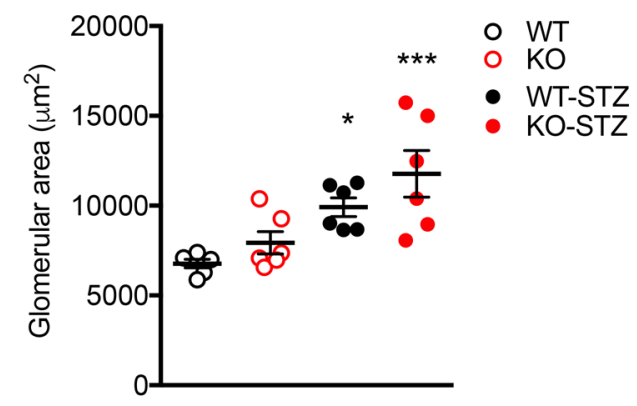

E

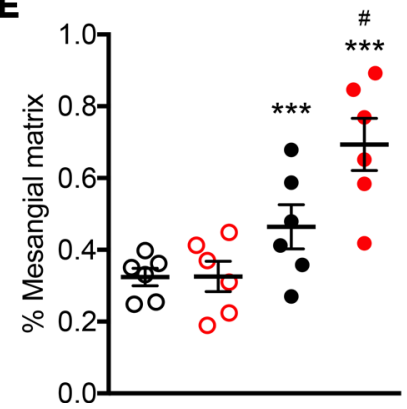

KO-STZ
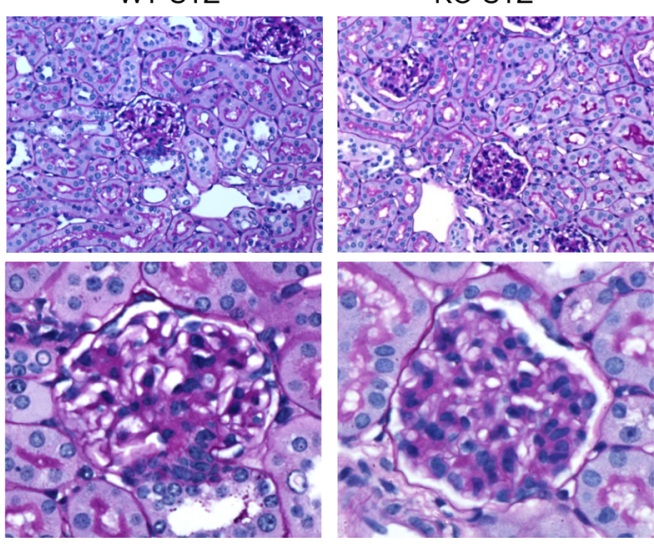

- WT

O KO

- WT-STZ

- KO-STZ

Figure 3. Global KO of Tyro3 increases albuminuria, glomerular hypertrophy, and mesangial expansion in diabetic mice. (A) Urinary albumin/creatinine ratio $(\mu \mathrm{g} / \mu \mathrm{g})$ in control WT, control Tyro3 KO, diabetic WT (WT-STZ), and diabetic Tyro3 KO (KO-STZ). (B) Twelve-hour albumin excretion in control and diabetic mice. (C) Periodic acid-Schiff-stained kidney images at 200x and 400x magnifications. Scale bar: $50 \mu \mathrm{m}$. (D and E) Quantification of glomerular area (D) and percentage of mesangial matrix $(\mathbf{E})$ in control and diabetic mice. For all datasets, $n=6$ in each group; data are shown as mean \pm SEM; statistical analysis was performed with 2-way (A) or 1-way ANOVA (B, D, and E) with Tukey's multiple comparison test. ${ }^{*} P<0.05$, ${ }^{* *} P<0.01$, ${ }^{* *} P<0.001$, and ${ }^{* * *} P<0.0001$ when compared with nondiabetic control mice; ${ }^{*} P<0.05,{ }^{* \#} P<0.01$, \#\#\# $P<0.001$ when compared with WT-STZ mice.

in Tg26 mice abated albuminuria, glomerulosclerosis, and ensuing renal fibrosis (Supplemental Figure 6). Together with the above findings in DKD, these results strongly indicate the protective effects of TYRO3 against podocyte injury and suggest that TYRO3 could be a potential therapy to ameliorate podocyte injury and glomerular disease progression.

Regulation of TYRO3 in progressive kidney disease. The relative increase in TYRO3 levels observed in the early stage of DKD appears to be diminished in progressive DKD. Our previous findings demonstrated that high glucose could upregulate both PS and TYRO3 expression in cultured podocytes (7). Interestingly, the increase in TYRO3 expression in diabetic kidneys is lost in podocyte-specific PS-KO mice (Supplemental Figure 7), suggesting a positive feedback loop of TYRO3 expression by signal transduction through its ligand PS. To determine the potential mechanism of TYRO3 suppression in progressive DKD, we performed in silico promoter analysis of TYRO3, which identified several potential binding sites for NF- $\mathrm{KB}$. We confirmed in cultured podocytes that TNF- $\alpha$ stimulation suppressed 
A

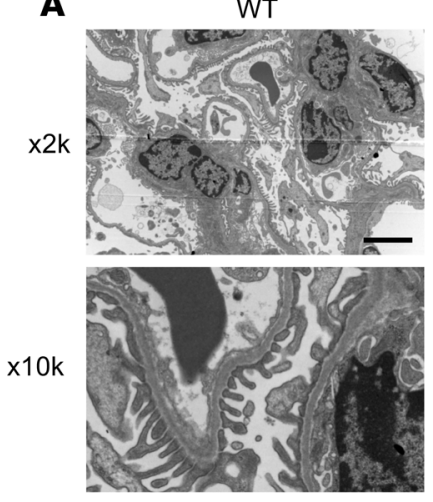

B

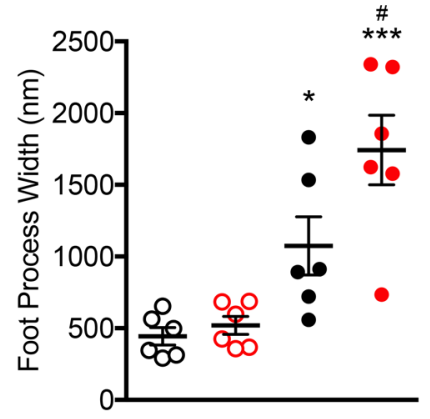

D

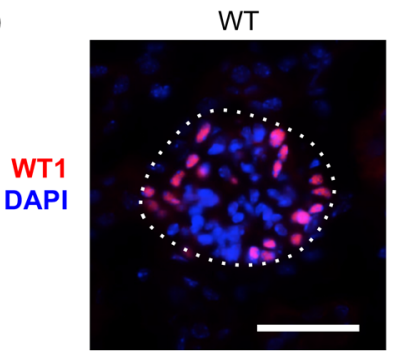

KO

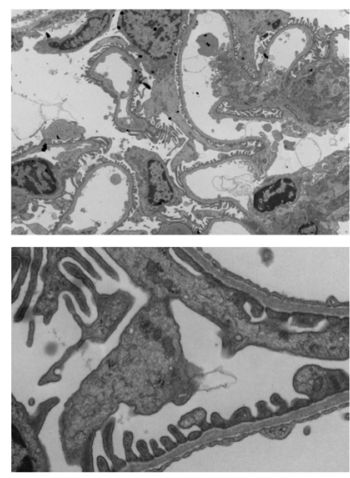

$\begin{array}{llll}\# & & & \\ * * * & \circ & W T\end{array}$

- KO

- WT-STZ

- KO-STZ
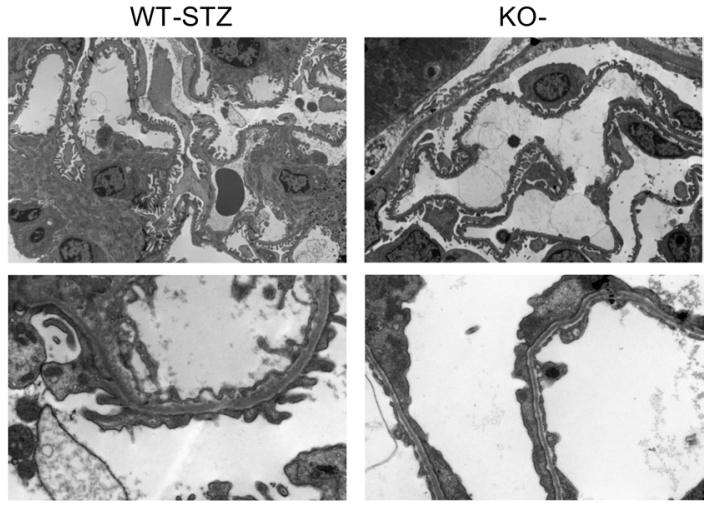

C

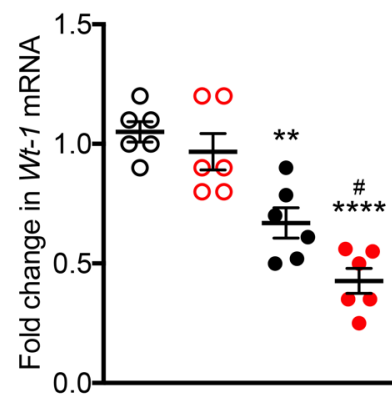

O WT

○ KO

- WT-STZ

- KO-STZ

KO
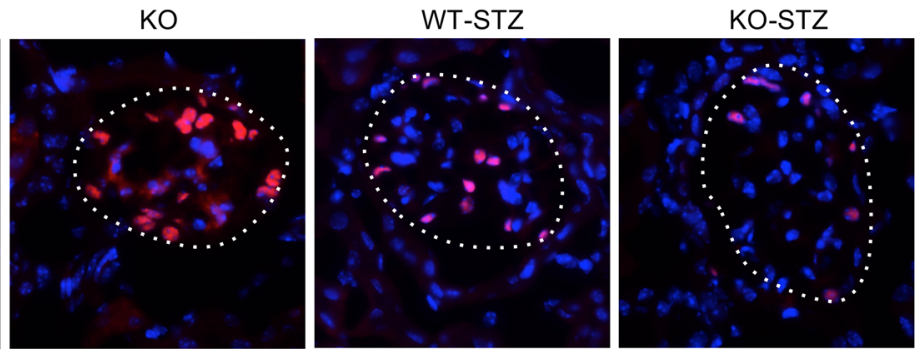

E

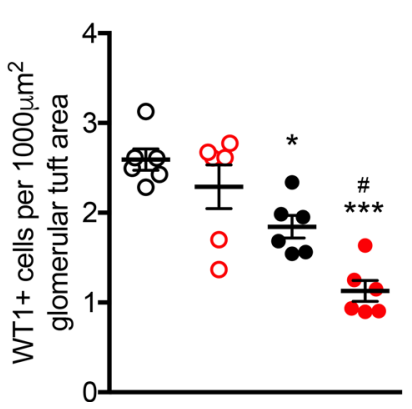

○ WT

- KO

- WT-STZ

- KO-STZ

Figure 4. Global KO of TyrO3 increases podocyte foot process effacement and loss in diabetic mice. (A) Representative transmission EM images of control and diabetic WT and Tyro3 KO mice at 2,000 $\times(\times 2 \mathrm{k})$ and 10,000× (×10k) magnifications. Scale bar: $5 \mu \mathrm{m}$. (B) Quantification of foot process width (nm) in control and diabetic mice. (C) Quantitative PCR analysis of WT1 mRNA in isolated glomeruli from control and diabetic mice. (D) Representative images WT1 immunofluorescence. Glomeruli are outlined with dotted lines. Scale bar: $50 \mu \mathrm{m}$. (E) Number of WT1 $1^{+}$cells per 1, $000 \mu \mathrm{m}^{2}$ glomerular tuft area. Data are shown as mean \pm SEM; $n=6$ in each group; statistical analysis was performed 1-way ANOVA with Tukey's multiple comparison test. ${ }^{*} P<0.05$, ${ }^{* *} P<0.01$, ${ }^{* *} P<0.001$, and ${ }^{* * *} P<0.0001$ when compared with nondiabetic control mice; ${ }^{*} P<0.05$ when compared with WT-STZ mice.

TYRO3 expression and that this effect was alleviated by NF-kB inhibitor BAY 11-7082 (Figure 10, A and B). As NF-KB is a key mediator of progressive DKD (26-28) and of other glomerular diseases such as HIVAN (29), the suppression of TYRO3 expression in progressive kidney disease may be mediated, in part, through the activation of the NF-kB pathway.

Role of TYRO3 in podocyte apoptosis. We observed that the global loss of Tyro3 exacerbated podocyte injury and lossinDKD and in ADRN, whereaspodocyte-specific overexpression of Tyro3attenuated them. 
A

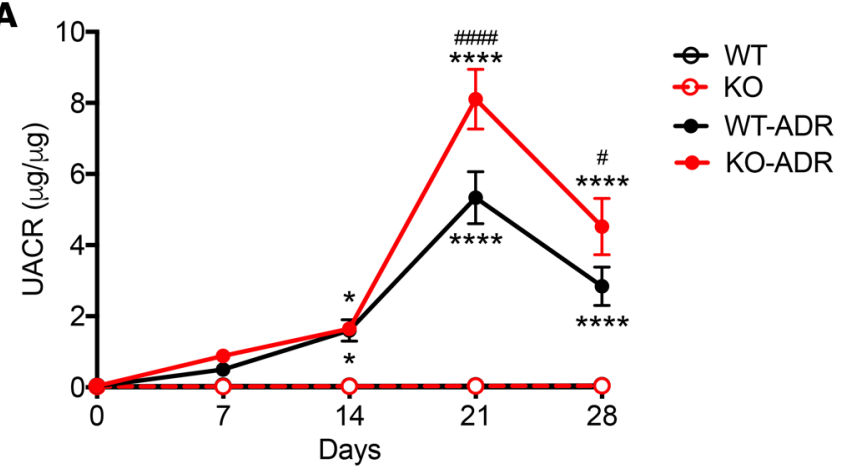

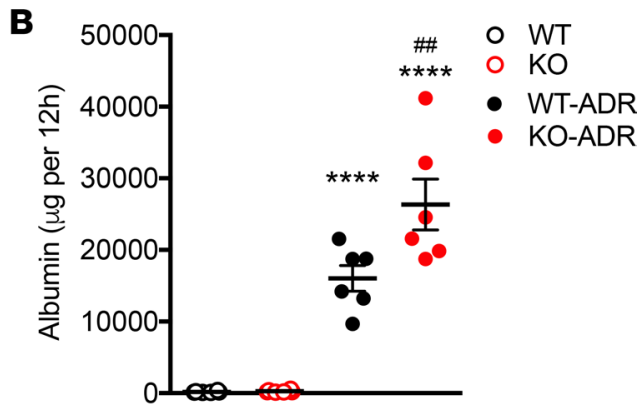
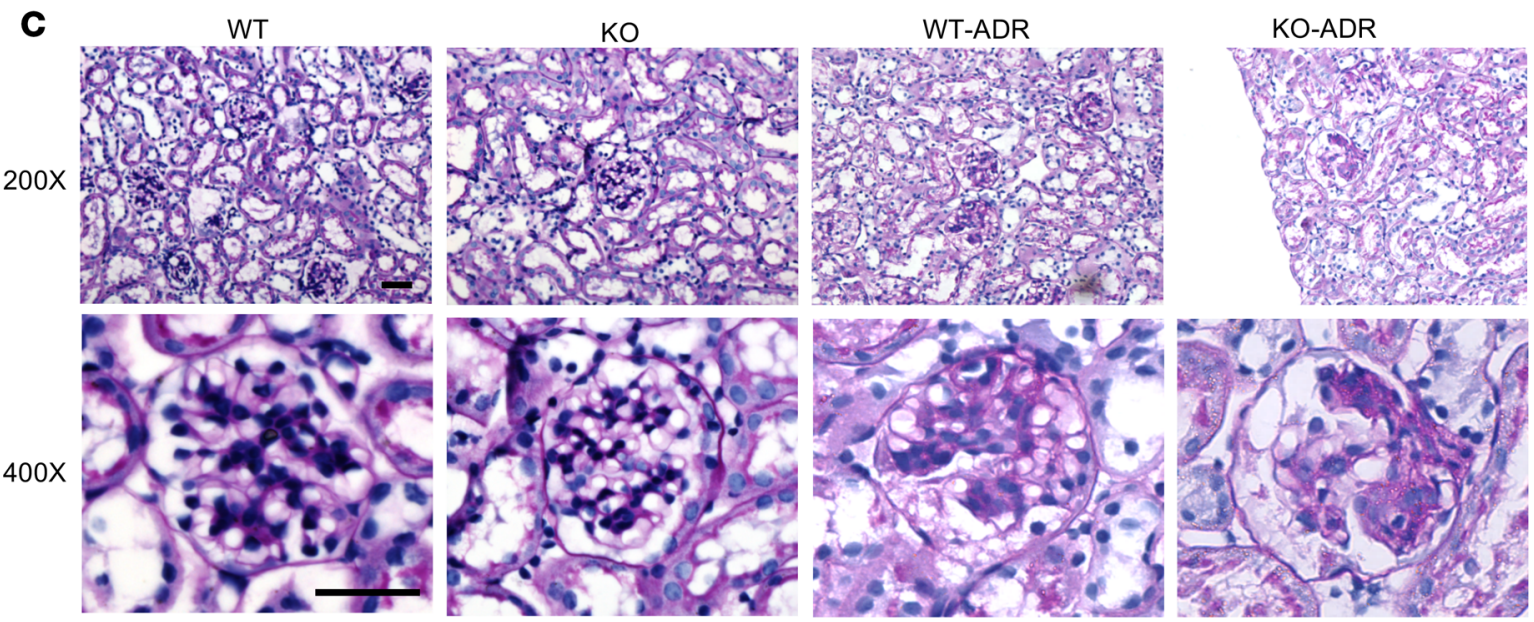

D

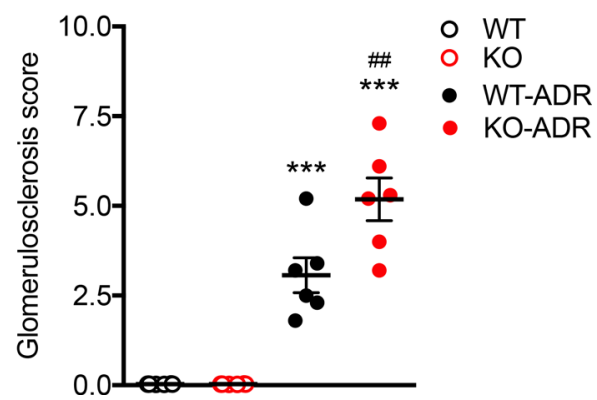

Figure 5. Global knockout of Tyro3 increases albuminuria and glomerulosclerosis in ADRN mice. (A) Urinary albumin/creatinine ratio ( $\mu$ g/ $\mu$ g) in control WT, control Tyro3 KO (KO), ADR-injected WT (WT-ADR), and ADR-injected Tyro3 KO (KO-ADR). (B) Twelve-hour albumin excretion ( $\mu$ g) in control and ADRN mice. (C) Periodic acid-Schiff-stained kidney images at 200x and 400x magnifications. Scale bar: $50 \mu \mathrm{m}$ (D) Semiquantification of glomerulosclerosis in control and ADRN mice. For all datasets, $n=6$ in each group; data are shown as mean \pm SEM; statistical analysis was performed with 2-way $(\mathbf{A})$ or 1 -way ANOVA (B and $\mathbf{D})$; ${ }^{*} P<0.05,{ }^{* *} P<0.001$, and ${ }^{*}{ }^{*} P<0.0001$ when compared with vehicle-injected control mice;

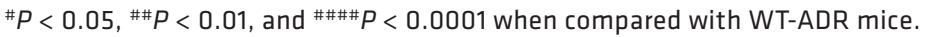

One of the downstream signaling mediators of TYRO3 is AKT (30). In cultured podocytes, TYRO3 ${ }^{\text {OV }}$ led to enhanced AKT phosphorylation, whereas TYRO3 ${ }^{\mathrm{KD}}$ diminished it (Figure 10C). As AKT activation protects podocytes from apoptosis-induced cell death (31), we next determined the effects of TYRO3 overexpression and knockdown on podocyte apoptosis. TYRO3 ${ }^{\mathrm{KD}}$ increased podocyte apoptosis by annexin V staining (Figure 10, D and E). Moreover, the increase in podocyte apoptosis, when challenged with high glucose conditions, was mitigated with TYRO3 overexpression, whereas it was enhanced with TYRO3 ${ }^{\mathrm{KD}}$ (Figure 10F). Similarly, supplementation of PS (10 nM) decreased podocyte apoptosis, while this protection was lost with the inhibition of AKT with a specific AKT inhibitor (AKTi; $5 \mu \mathrm{M}$ ) (Figure 10G). Together, these results suggest that PS/TYRO3 signal transduction protects against podocyte injury and loss through the activation of the AKT pathway and enhancing their survival. 

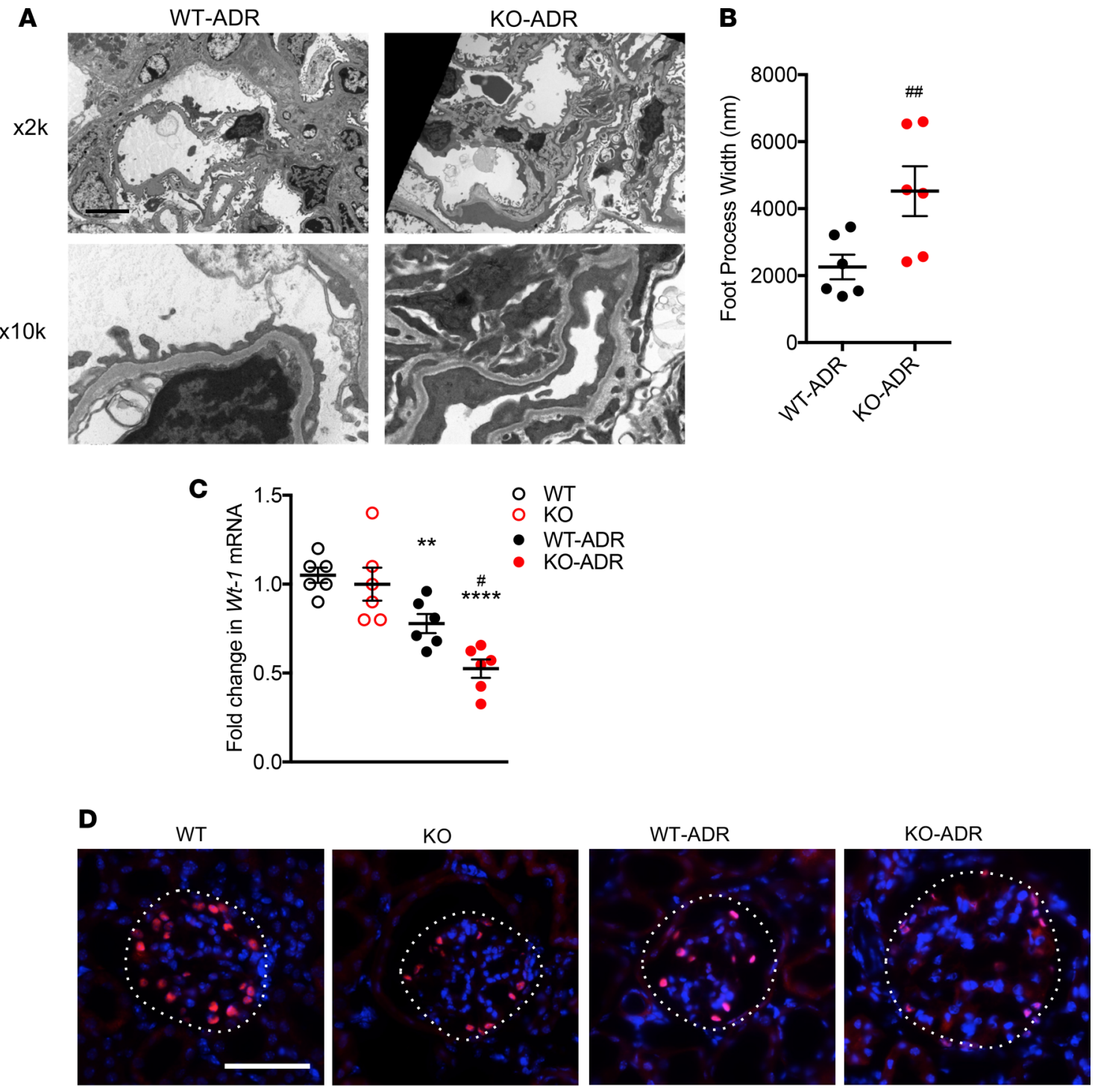

E

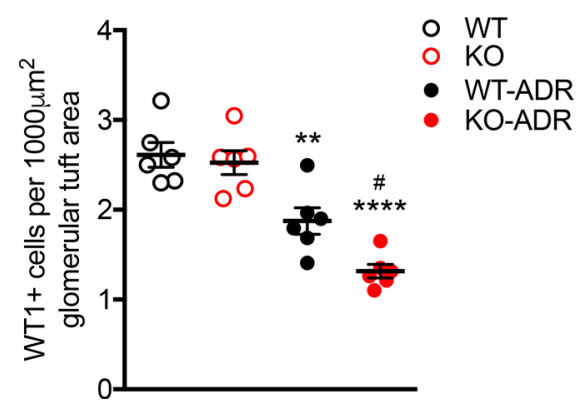

Figure 6. Global KO of Tyro3 increases podocyte foot process effacement and loss in ADRN mice. (A) Representative transmission EM images of control and ADRN mice at 2,000× $(\times 2 \mathrm{k})$ and $10,000 \times(\times 10 \mathrm{k})$ magnifications. Scale bar: $5 \mu \mathrm{m}$. (B) Quantification of foot process width (nm) in control and ADRN mice. One-tailed, paired $t$ test. (C) Quantitative PCR analysis of WT1 mRNA in isolated glomeruli from control and ADRN mice. (D)

Representative images WT1 immunofluorescence. Scale bar: $20 \mu \mathrm{m}$. (E) Number of WT1+ cells per 1,000 $\mu \mathrm{m}^{2}$ glomerular tuft area. Data are shown as mean $\pm \mathrm{SEM} ; n=6$ in each group; statistical analysis was performed with 1-way ANOVA with Tukey's multiple comparison test; ${ }^{* *} P<0.01$ and ${ }^{* * *} P<0.0001$ when compared with vehicle-injected control mice; ${ }^{*} P<0.05$ and ${ }^{\# \#} P<0.01$ when compared with WT-ADR. 


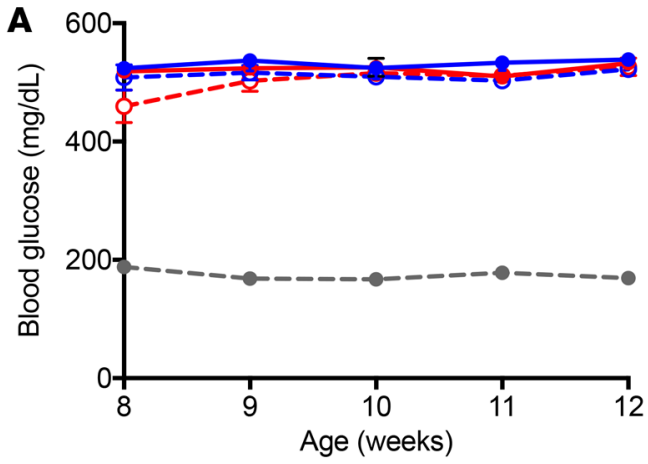

B

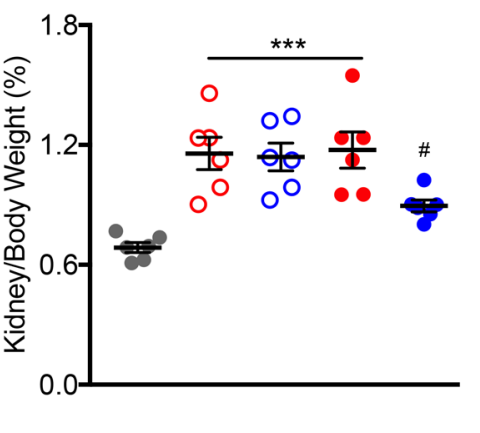

O OVE26
OVE26;Tyro3 OV ]-DOX

- OVE26 ${ }_{\text {- OVE26;Tyro3 }}$ OV]+DOX

- WT
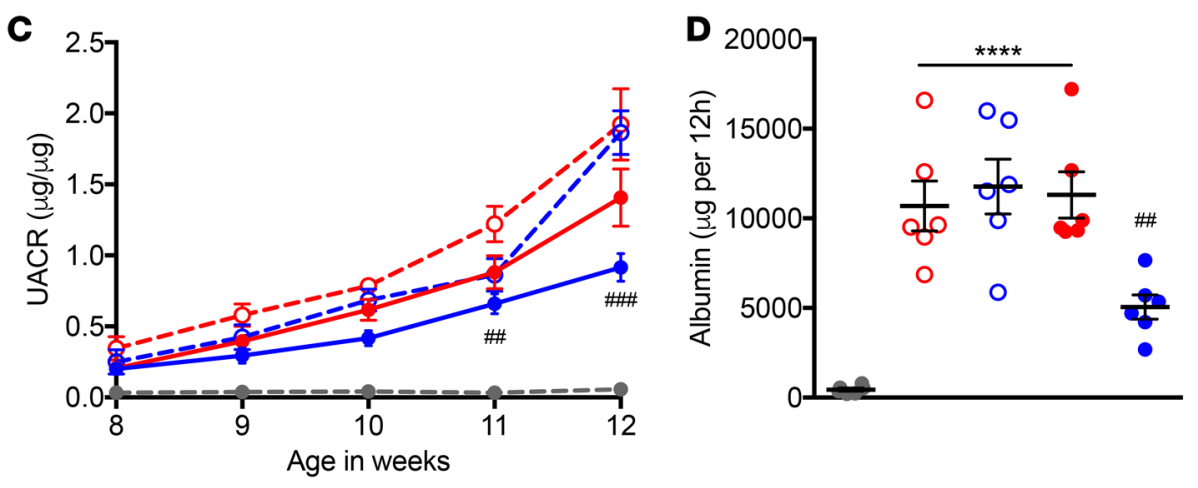

$\begin{array}{ll}\circ & \text { OVE26 } \\ \circ & \text { OVE26;Tyro3 } \\ & \text { OV ]-DOX }\end{array}$

- OVE26

- WT

E

- DOX

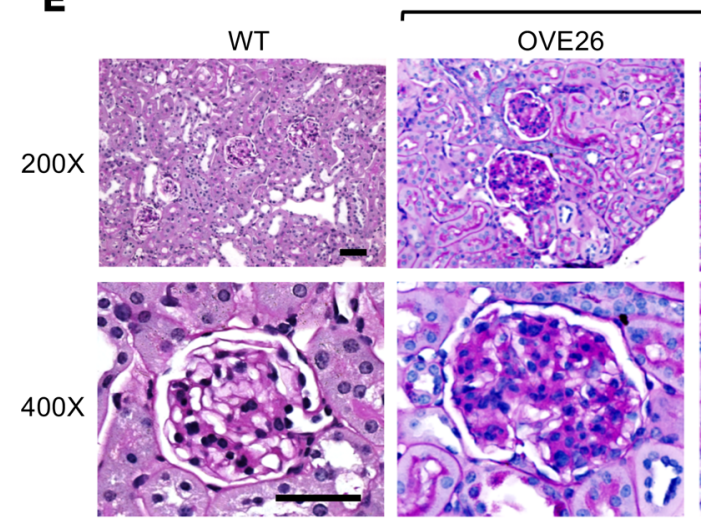

OVE26;Tyro3 ${ }^{\circ}$

+ DOX

OVE26;Tyro3ov
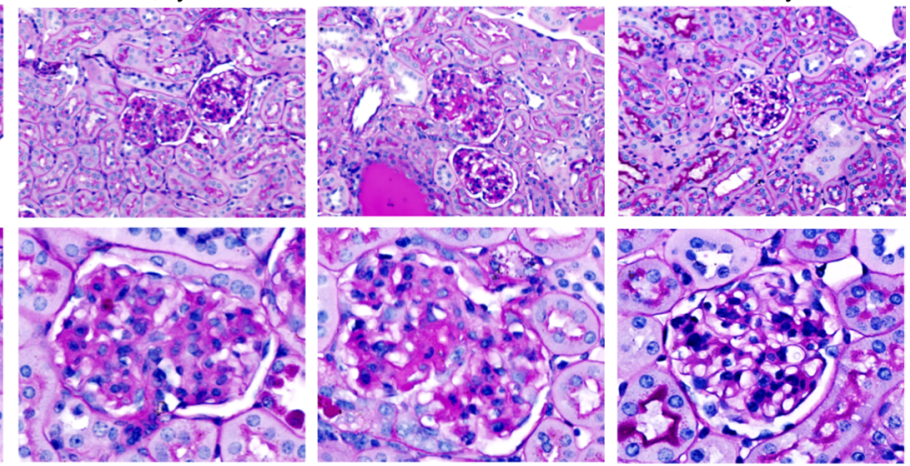

$\mathbf{F}$

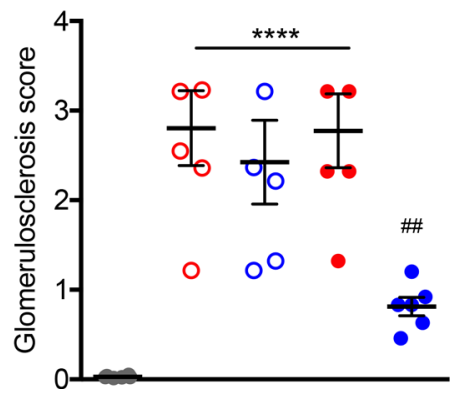

$\begin{array}{ll}\text { O } & \text { OVE26 } \\ \text { O } & \text { OVE26;Tyro3OV ]-DOX }\end{array}$

- OVE26

- OVE26;Tyro3 $\left.{ }^{O V}\right]+D O X$

- WT
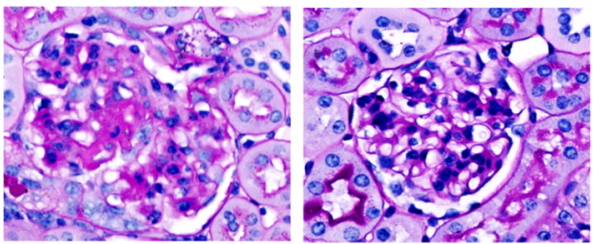

Figure 7. Podocyte-specific overexpression of Tyro3 ameliorated albuminuria, glomerular hypertrophy, and mesangial expansion in diabetic mice. (A) Blood glucose levels in control WT, control OVE26 and OVE26;Tyro3 ${ }^{\circ \mathrm{V}}$ mice, and DOX-treated OVE26 and OVE26;Tyro3 ${ }^{\circ \mathrm{V}}$ mice. (B) Kidney/body weight ratio in control and diabetic mice. (C) Urinary albumin/creatinine ratio (UACR) in control and diabetic mice. (D) Twelve-hour albumin excretion in control and diabetic mice. (E) Periodic acid-Schiff stained kidney images at 200x and 400x magnifications. Scale bar: $50 \mu \mathrm{m}$. (F) Quantification of glomerulosclerosis scoring in control and diabetic mice. For all datasets, $n=6$ in each group; data are shown as mean \pm SEM; statistical analysis was performed with 2-way (A and $\mathbf{C}$ ) or 1-way ANOVA (B and $\mathbf{D})$ with Tukey's multiple comparison test. ${ }^{* *} P<0.001$ and ${ }^{* * * *} P<0.0001$ when compared with WT mice; ${ }^{\#} P<0.05,{ }^{\# \#} P<0.01$, and ${ }^{\# \#} P<0.001$ when compared with control OVE26 mice. 
A

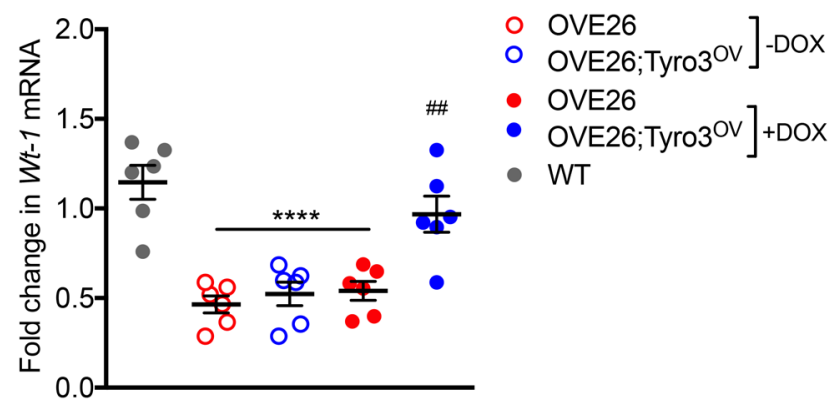

B
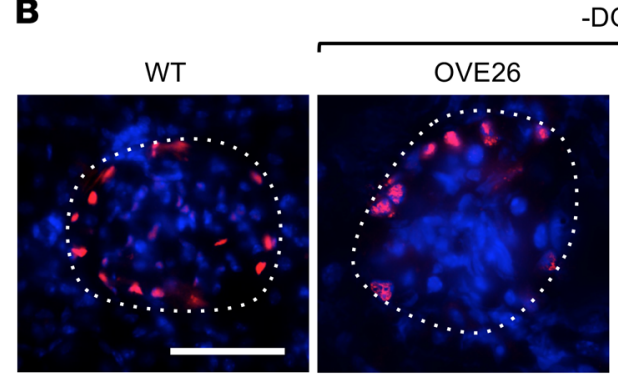

DOX

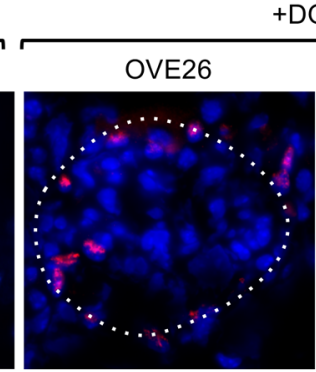

$+\mathrm{DOX}$

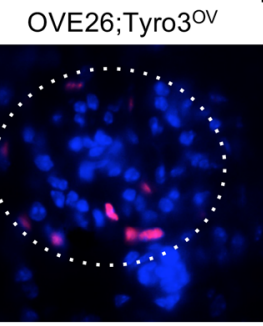

\section{OVE26;Tyro3 ${ }^{\text {OV }}$}

\section{C}
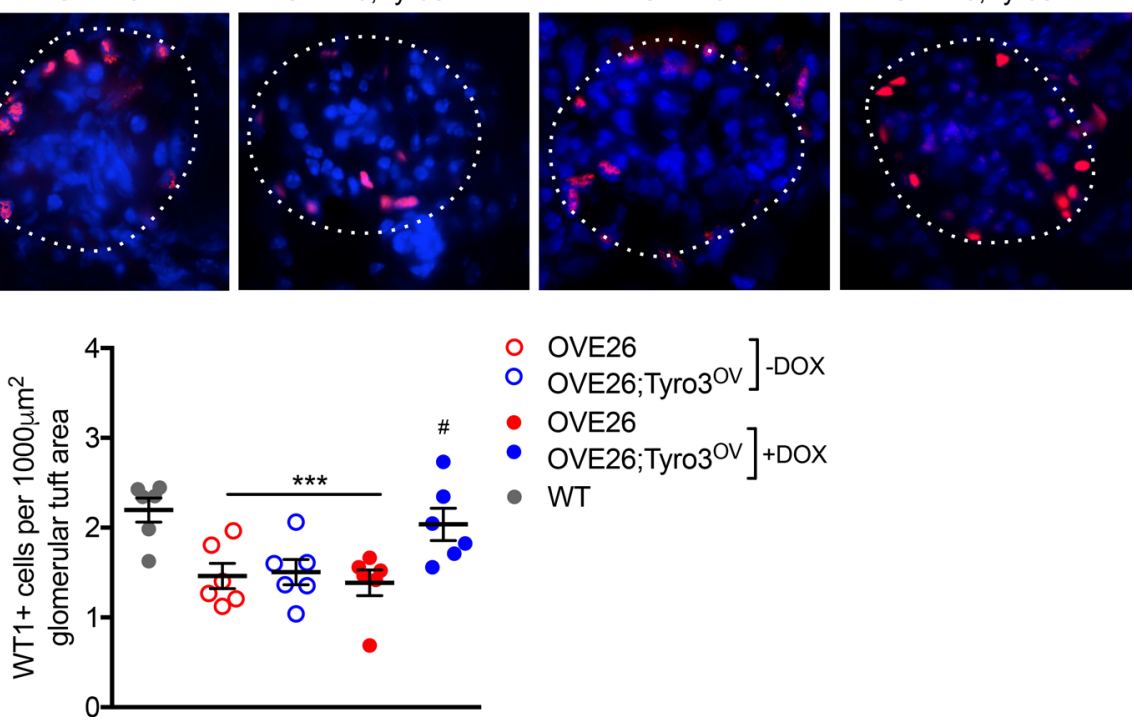

Figure 8. Podocyte-specific overexpression of Tyro3 ameliorates podocyte loss in diabetic mice. (A) Quantitative PCR analysis of WT1 mRNA in isolated glomeruli from control and diabetic mice. (B) Representative images WT1 immunofluorescence. (C) Number of WT1+ ${ }^{+}$cells per $1,000 \mu \mathrm{m}^{2} \mathrm{glomerular}$ tuft area. Data are shown as mean $\pm \mathrm{SEM} ; n=6$ in each group; statistical analysis was performed 1-way ANOVA with Tukey's multiple comparison test. ${ }^{* * *} P<$ 0.001 and ${ }^{* * *} P<0.0001$ when compared with $\mathrm{WT} ;{ }^{*} P<0.05$ and ${ }^{\# \#} P<0.01$ when compared control OVE26 mice.

\section{Discussion}

Podocyte injury is a major event in both primary and secondary glomerular disease. Loss of podocytes by either apoptosis or detachment or by other mechanisms leads to progression of glomerular disease (1, 2, 5). Of all glomerular morphological characteristics, the reduction in podocyte density is considered as the strongest predictor of progressive $\operatorname{DKD}(32,33)$, and the degree of podocyte reduction correlates directly with the magnitude of proteinuria $(34,35)$. Many studies have identified more specific treatments to prevent podocyte injury. For example, several immunosuppressive medications, such as calcineurin inhibitors, steroids, and rituximab, have direct protective effects against podocyte injury (36-38). We and others have showed that retinoic acid could attenuate podocyte injury in glomerular disease (39). However, clinical trials with retinoids are limited because of their significant side effects. Vitamin D3 analogues also reduce podocyte injury in glomerular disease, but the effects have not been tested by clinical trials (40). Our data now demonstrate that TYRO3 could be a potential target to treat podocyte injury in glomerular disease.

We found that TYRO3 expression was localized mostly in podocytes in human kidneys and that its expression was markedly suppressed in the glomeruli of patients with progressive DKD and FSGS. Interestingly, TYRO3 expression was increased in the glomeruli of type 2 diabetic Pima Indians with early and mild DKD (41), but it was significantly decreased in glomeruli of White subjects with progressive DKD (42). A correlation between eGFR and glomerular TYRO3 expression was also observed in DKD patients, indicating an association of TYRO3 expression with the disease progression. As mouse models of DKD display a milder disease phenotype than displayed in human DKD and mimic more of early diabetic kidneys (43), the observation that Tyro3 mRNA expression is increased in $d b / d b$ or STZ-induced 
A

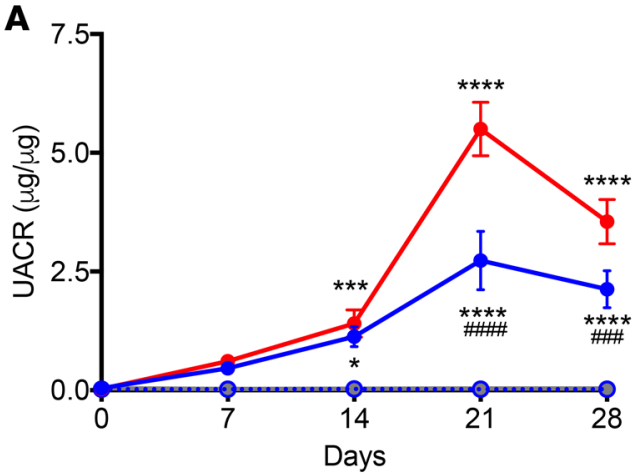

B

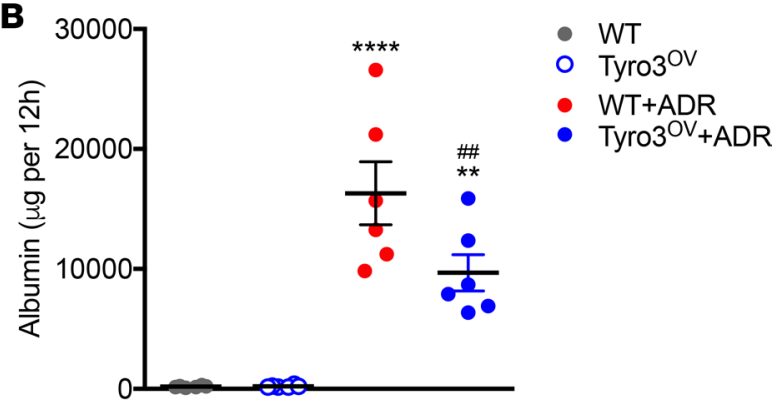

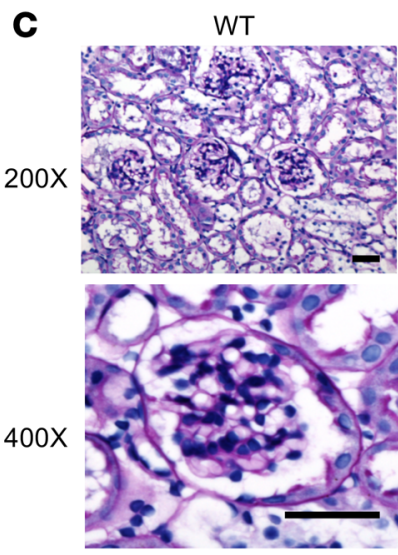
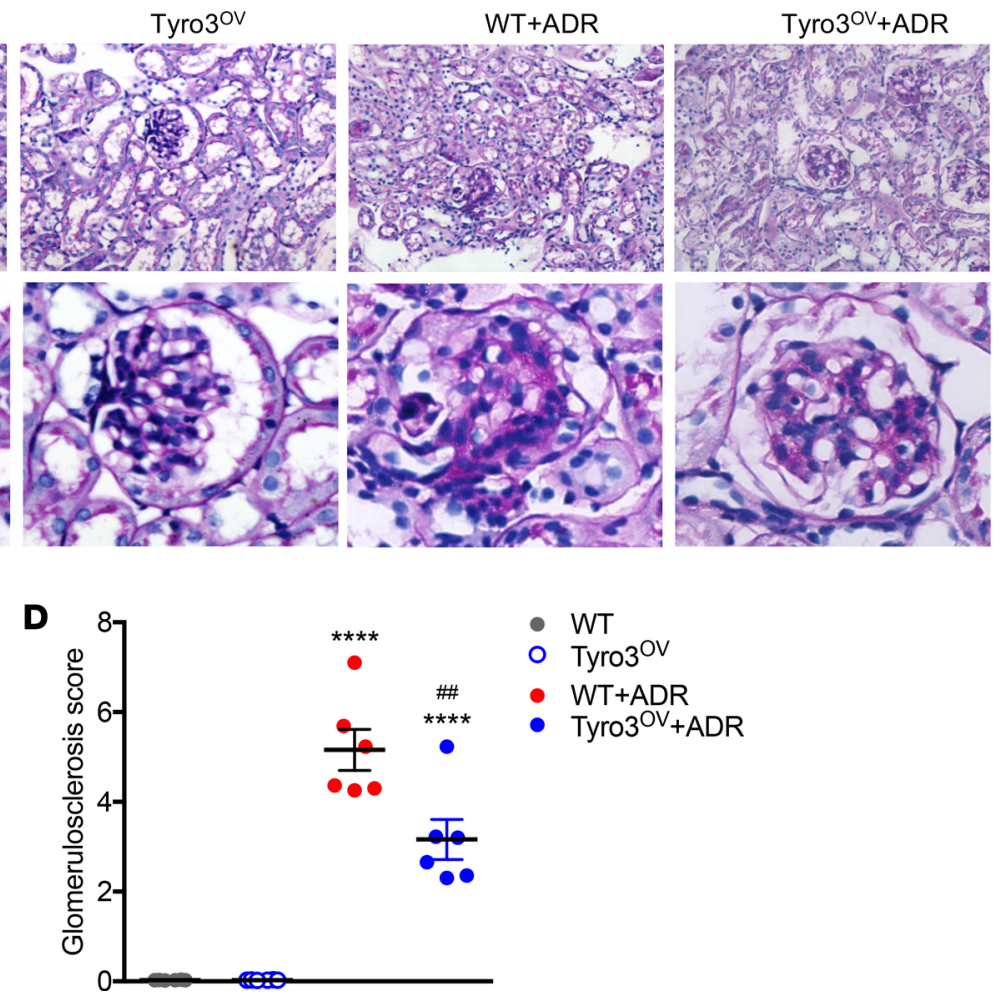

Figure 9. Podocyte-specific overexpression of Tyro3 ameliorates albuminuria and glomerulosclerosis in ADRN mice. (A) Urinary albumin/creatinine ratio in DOX-treated control WT, Tyro3 ${ }^{\mathrm{OV}}$, ADR-injected WT (WT+ADR), and ADR-injected Tyro3 ${ }^{\mathrm{OV}}$ (Tyro3 $\left.{ }^{\mathrm{OV}}+\mathrm{ADR}\right)$. (B) Twelve-hour albumin excretion in control and ADRN mice. (C) Periodic acid-Schiff-stained kidney images at 200x and 400x magnifications. Scale bar: $50 \mu$ m. (D) Semiquantification of glomerulosclerosis in control and ADRN mice. For all datasets, $n=6$ in each group; data are shown as mean $\pm S E M$; statistical analysis was performed with 2-way (A) or 1-way ANOVA (B and D) with Tukey's multiple comparison test; ${ }^{* *} P<0.01,{ }^{* *} P<0.001$, and ${ }^{* * *} P<0.0001$ when compared with vehicle-injected

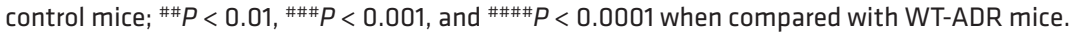

diabetic mice (43) - but mildly suppressed in glomeruli of eNOS-deficient $d b / d b$ mice with more progressive kidney disease - is consistent with the findings in human kidneys. We previously showed that the expression of both PS and TYRO3 are increased by high glucose conditions in vitro (7), suggesting that their upregulation in early DKD may be a protective mechanism against cellular injury in the diabetic milieu and that the renoprotective mechanism from PS/TYRO3 is lost in patients with progressive DKD Although the exact mechanisms of PS and TYRO3 downregulation in the late stage of DKD remain to be elucidated, our in vitro data suggest that the activation of the TNF- $\alpha / \mathrm{NF}-\mathrm{kB}$ pathway, which plays a critical role in the progression of DKD and other glomerular diseases $(27,44)$, is in part responsible for the reduced TYRO3 expression in patients with progressive kidney disease.

Our in vitro data also indicate that TYRO3 signaling is important for podocyte survival through the activation of the AKT pathway, a prosurvival pathway in podocytes in early DKD $(45,46)$. Knockout of Tyro3 indeed exacerbated podocyte loss and DKD progression in STZ-induced diabetic mice. 
A

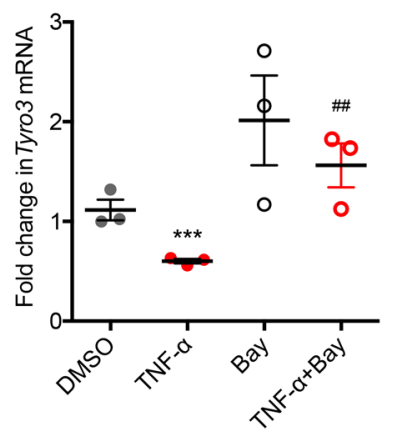

B

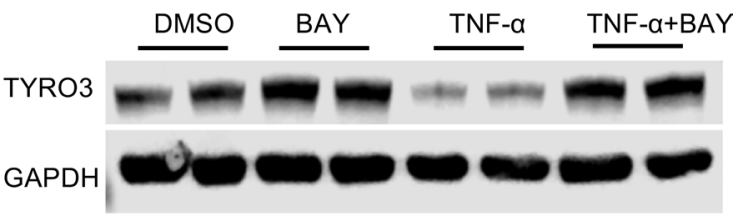

Figure 10. TYRO3 signaling affects AKT activation in cultured podocytes. (A) Real-time PCR analysis of TYRO3 mRNA in cultured human podocytes after TNF- $\alpha$ treatment (10 $\mathrm{ng} / \mathrm{ml}$ ) for 6 hours with or without pretreatment with NF- $\kappa B$ inhibitor BAY11-7082 (BAY, $5 \mu$ M) for 1 hour. $n$ $=3$ per sample; 2 -tailed, unpaired $t$ test was performed to compare means between groups. ${ }^{* *} P<0.001$ when compared with DMSO control, ${ }^{\# P} P<$ 0.01 when compared with TNF- $\alpha$. (B) Western blot analysis of TYRO3 in cells in A. (C) Western blot analysis of total and phosphorylated AKT in cultured podocytes with Tyro3 overexpression (Tyro3 ${ }^{\mathrm{OV}}$ ) or Tyro3 knockdown (Tyro $3^{\mathrm{KD}}$ ) compared with control knockdown (Control ${ }^{\mathrm{KD}}$ ) podocytes. (D and E) Annexin $\mathrm{V}$ analysis of apoptosis in Control ${ }^{\mathrm{KD}}$ and $\mathrm{Tyro}^{\mathrm{KD}}$ podocytes. FACS analysis is shown in $\mathbf{D}$, and quantification is shown in $\mathbf{E}$. $n=4$ per sample; 2 -tailed, unpaired $t$ test was performed to compare means between groups. ${ }^{* *} P$ $<0.001$ when compared with Control ${ }^{\mathrm{KD}}$. (F) Active Caspase-3 concentration in cultured podocytes (WT, Tyro3 ${ }^{\circ}$, or Tyro3 ${ }^{\mathrm{KD}}$ ) under normal (control), high mannitol (mannitol), or high glucose (glucose) conditions. $n=5$; 1 -way ANOVA with Tukey's multiple comparison test was performed to compare means. ${ }^{* * * *} P<0.0001$ when compared with control group; $\# P<0.01$ and $\# \# \# \#<$ 0.0001 when compared with glucose group. (C) Active Caspase-3 concentration in cultured podocytes under normal (control), high mannitol (mannitol), or high glucose (glucose) conditions with or without protein $S$ (PS; $50 \mathrm{nM}$ ) and $A K T$ inhibitor (AKTi; $1 \mu \mathrm{M}) . n=5$; 1-way ANOVA with Tukey's multiple comparison test was performed to compare means. ${ }^{* * *} P<0.0001$ when compared with control group; $\# P<0.01$ when compared with glucose group; $\$ \$ \$ p<0.0001$ when compared with glucose+PS group.

While we cannot preclude the effects of Tyro3 loss in cells outside the kidney in contributing to the worsened DKD phenotype in diabetic Tyro3-null mice, podocyte-specific overexpression of Tyro3 was sufficient to significantly attenuate podocyte loss and DKD injury in the OVE26 model of type 2 diabetes, confirming an important podocyte-specific role of TYRO3 in diabetic kidneys. As other glomerular cell injuries are involved in diabetic glomerulopathy in addition to podocytes, we expect that therapeutic targeting of TYRO3 in combination with other therapies, such as renin-angiotensin blockade, may be optimal as a potential DKD treatment.

In addition to $\mathrm{DKD}$, decreased expression of TYRO3 was also observed in glomeruli of patients with FSGS, and it correlated with the progression of primary glomerular diseases, as observed in the NEPTUNE study. Our data using Tyro3-KO and podocyte-specific Tyro3 overexpression transgenic mice 
indicates the renoprotective role of TYRO3 not only in DKD, but also in ADRN and HIVAN. Together, our data underline the previously unrecognized role of TYRO3 as an important mediator of podocyte survival and a potential drug target for treatment in glomerular disease.

\section{Methods}

Gene expression analysis in human kidney tissues. Human kidney tissue (glomeruli or tubuli) was microdissected as described previously (27). Expression of TYRO3 in microdissected glomeruli from 128 adult patients from NEPTUNE and 22 Southwestern American Pima Indians with early stage of DKD was extracted from Affymetrix GeneChip array analysis $(43,47,48)$.

IHC. Briefly, formalin-fixed and paraffin-embedded sections were deparaffinized, and endogenous peroxidase was inactivated with $\mathrm{H}_{2} \mathrm{O}_{2}$. Sections were then blocked in $2 \%$ goat serum in PBS for 1 hour at room temperature and then incubated with primary antibody at $4^{\circ} \mathrm{C}$ overnight. The next day, sections were washed 3 times with PBS and then incubated with secondary antibody for 30 minutes. Positive staining was revealed by peroxidase-labeled streptavidin and diaminobenzidine substrate with a fixed exposure time of 3 minutes for all experiments among the groups. The control included a section stained with only secondary antibody (Vectastain ABC kit, pk-1601, Vector Laboratories).

Quantification of Immunostaining. Immunostained images with magnification of $400 \times$ were obtained. ImageJ (NIH) 1.26t software was used to measure the level of immunostaining in the glomeruli. First, the images were converted to 8-bit gray scale. Glomerular regions were selected for measurement of area and integrated density, and background intensity was measured by selecting 3 distinct areas in the background with no staining. The corrected optical density (COD) was determined as shown below:

$\mathrm{COD}=\mathrm{ID}-(\mathrm{A} \times \mathrm{MGV})$,

where ID is the integrated density of the selected glomerular region, A is the area of the selected glomerular region, and MGV is the mean gray value of the background readings (49).

Diabetic mouse models. Global Tyro3 ${ }^{-1-}$ mice in C57BL/6J background were purchased from The Jackson Laboratory (strain no. 007937). The inducible podocyte-specific Tyro3 overexpression mouse model (Tyro3 ${ }^{\mathrm{OV}}$ ) was generated at the Mouse Genetics and Gene Targeting Core at Icahn School of Medicine at Mount Sinai. At 8 weeks of age, Tyro $^{+/+}$and Tyro $3^{-/-}$male mice were i.p. injected with either STZ ( $50 \mu \mathrm{g} / \mathrm{g}$; MilliporeSigma) or sodium citrate vehicle for 5 consecutive days. Fasting blood glucose concentrations were monitored weekly by using a glucometer. Mice were sacrificed at 20 weeks after STZ injection. Diabetic OVE26 mice (strain no. 005564; The Jackson Laboratory) were crossed with inducible podocyte-specific Tyro3 overexpression mice (podocin-rtTA;TetON-Tyro3) in the FVB/N background to generate OVE26; Tyro3 ${ }^{\mathrm{OV}}$ mice. Doxycycline-supplemented chow (635 mg/kg; Envigo RMS Inc.) was given at 8 weeks of age and mice were sacrificed at 12 weeks of age. Age-matched OVE26 mice and WT FVB/N mice were used as controls. Diabetic OVE26 mice were maintained by daily monitoring of blood glucose and long-acting insulin injections as needed.

ADRN and HIVAN murine models. ADRN was induced by injection of $18.8 \mathrm{mg} / \mathrm{kg}$ adriamycin via the tail vein in Tyro $^{\mathrm{OV}}$ and their control littermates at 10 weeks of age, as described previously (50). Proteinuria was monitored every week, and the mice were sacrificed at 28 days after injection. Tyro ${ }^{\text {OV }}$ mice were also crossed with HIV-1 transgenic mice (Tg26) in the FVB/N background. Tyro ${ }^{\mathrm{OV}} ; \mathrm{Tg} 26$ mice were compared with Tg26 mice or WT normal mice in FVB/N background. The proteinuria was monitored weekly in these mice starting at 4 weeks of age, and the mice were sacrificed at a 8 weeks of age.

Measurement of urine albumin and creatinine. Urine albumin was quantified by ELISA using a kit from Bethyl Laboratories Inc. Urine creatinine levels were measured in the same samples using QuantiChrom creatinine assay kit (DICT-500; BioAssay Systems) according to the manufacturer's instruction. The urine albumin excretion was assessed as the ratio of albumin to creatinine. Twelve-hour urine collections in the metabolic cages were also used for determination of the urinary albumin excretion rate.

Kidney histology. Kidneys were removed and fixed with $4 \%$ paraformaldehyde for 16 hours at $4^{\circ} \mathrm{C}$. The 4- $\mu \mathrm{m}$ sections were cut from paraffin-embedded kidney tissues. Sections were stained with periodic acid-Schiff (PAS) for histology analysis. Assessment of the mesangial and glomerular cross-sectional areas was performed in a blinded fashion by pixel counts on a minimum of 10 glomeruli per section, under 400× magnification (Zeiss AX10 microscope). Renal histological abnormalities were scored as previously described (51). The glomerulosclerosis was graded on a semiquantitative scale (0 to $3+$ ): 0 (absent), $1+$ (involving $1 \%-25 \%$ of all glomeruli sampled), $2+$ (involving $26 \%-50 \%$ of glomeruli), and $3+$ (involving $>50 \%$ of glomeruli) as described. An average of 50 glomeruli were sampled per animal. 
Electron microscopy. Tissues were fixed in $2.5 \%$ glutaraldehyde with $0.1 \mathrm{M}$ sodium cacodylate (pH 7.4) for 72 hours at $4^{\circ} \mathrm{C}$. Samples were further incubated for 1 hour at room temperature with $2 \%$ osmium tetroxide and $0.1 \mathrm{M}$ sodium cacodylate ( $\mathrm{pH}$ 7.4). Ultrathin sections were stained with lead citrate and uranyl acetate and viewed on a Hitachi $\mathrm{H} 7650$ microscope. Briefly, negatives were digitized, and images with a final magnitude of up to $10,000 \times$ were obtained. ImageJ $1.26 \mathrm{t}$ software was used to measure the length of the peripheral GBM, and the number of slit pores overlying this GBM length were counted. The arithmetic mean of the foot process width $\left(W_{\mathrm{FP}}\right)$ was calculated as shown below:

$W_{\mathrm{FP}}=\frac{\pi}{4} \times \frac{\sum G B M \text { LENGTH }}{\sum \text { silts }}$

where $\Sigma$ slits indicates the total number of slits counted; $\Sigma$ GBM LENGTH indicates the total GBM length measured in 1 glomerulus, and $\pi / 4$ is the correction factor for the random orientation by which the foot processes were sectioned (52).

Isolation of mouse glomeruli. Mouse glomeruli were isolated as described (53). Briefly, animals were perfused with HBSS containing $2.5 \mathrm{mg} / \mathrm{ml}$ iron oxide and $1 \%$ BSA. At the end of perfusion, kidneys were removed, decapsulated, minced into $1-\mathrm{mm}^{3}$ pieces, and digested in HBSS containing $1 \mathrm{mg} / \mathrm{ml}$ collagenase A and 100 units $/ \mathrm{ml}$ deoxyribonuclease I. Digested tissue was then passed through a $100-\mu \mathrm{m}$ cell strainer and collected by centrifugation ( $200 \mathrm{~g}$ for 5 minutes). The pellet was resuspended in $2 \mathrm{ml}$ of HBSS, and glomeruli were collected using a magnet. The purity of the glomerular collection was verified under microscopy. Total RNA was isolated from kidney glomeruli of mice using TRIzol (Invitrogen).

Cell culture. Human podocytes were obtained from Moin Saleem and cultured as described (54). Cells were serum starved in $1 \%$ serum containing medium for 12 hours, followed by treatment with the medium containing either normal glucose ( $5 \mathrm{mM}$ ), high mannitol ( $5 \mathrm{mM}$ glucose $\pm 25 \mathrm{mM}$ mannitol), or high glucose $(30 \mathrm{mM})$ for the indicated time intervals. Cells were serum starved in $1 \%$ serum containing medium for 12 hours. Cells were pretreated with or without NF-кB inhibitor (BAY 11-7082, $5 \mu \mathrm{M}$ ) for 1 hour in 1\% serum containing medium. The cells were then further stimulated with TNF- $\alpha$ (10 ng/ ml), human PS (hPS) (50 nM; Abnova, H00005627-P01), and/or AKT inhibitor (Tocris, 5773) $1 \mu \mathrm{M}$ for 24 hours as indicated.

Overexpression and knockdown PS and TAM receptors with shRNA-lentivirus. A Tyro3 clone was purchased from Thermo Fisher Scientific and inserted into a gag-, pol-, and env-deficient lentivector construct, $V V E / B B W$ (gift of G. Luca Gusella, Icahn School of Medicine at Mount Sinai). Lentiviral particles were generated and used for infection of podocytes. Podocytes with stable Tyro3 expression were selected using blasticidin. Cultured murine and human podocytes infected with the empty VVE/ $B B W$ lentivector served as controls. TYRO3 ${ }^{\mathrm{KD}}$ in human podocytes was performed using an Expression Arrest GIPZ Lentiviral shRNAmir system (Dharmacon). Two sets of shRNAs against hTYRO3 were used in the experiments. Lentiviral particles were produced by transfecting 293T cells with a combination of lentiviral expression plasmid, pCD/NL-BH $\Delta \Delta \Delta$ packaging plasmid, and VSV-G-encoding pLTR-G plasmid. For viral transduction of the human podocyte cell line, viral supernatants were supplemented with $8 \mu \mathrm{g} / \mathrm{ml}$ polybrene and incubated with cells for a 24 -hour period. Cells expressing shRNA were selected with puromycin for $2-3$ weeks prior to use in all studies. Western blot analysis was performed to confirm the efficiency of knockdown.

Western blot. Cells were homogenized in lysis buffer containing protease inhibitor cocktail. Equal amounts of protein samples were separated on SDS polyacrylamide gel, transferred to PVDF membranes (MilliporeSigma) and probed with primary antibodies (rabbit anti-TYRO3 from Abcam [ab109231], and rabbit anti-pAKT and anti-total AKT from Cell Signaling (catalogs 4060 and 9272). Membranes were then washed with PBST and incubated with a secondary antibody (horseradish peroxidase-conjugated antibodies to mouse IgG [Promega, W402B] or to rabbit IgG [Promega, W401B]). Blots were developed with the enhanced chemiluminescence system. Densitometry analysis for quantification was performed as described previously (55).

Real-time PCR. Total RNA was extracted by using TRIzol (Invitrogen). First-strand cDNA was prepared from total RNA $(2.0 \mu \mathrm{g})$ using the Superscript III first strand synthesis kit (Invitrogen), and cDNA (1 $\mu$ l) was amplified in triplicate using SYBR GreenER qPCR Supermix on an ABI PRISM 7900HT (Applied Biosystems). Light Cycler analysis software was used to determine crossing points using the second derivative method. Data were normalized to housekeeping genes (GAPDH) and presented as fold increase compared with RNA isolated from WT animals using the $2^{\Delta \Delta \mathrm{CT}}$ method. 
Immunofluorescence. Kidney sections from human kidney biopsies and from mice were immunostained using rabbit anti-Tyro3 (Abcam, ab109231), mouse antisynaptopodin (R\&D Systems, MAB8977), and mouse anti-WT1 (Santa Cruz Biotechnology Inc., sc-7385) antibodies. After washing, sections were incubated with a fluorophore-linked secondary antibody (Alexa Fluor 488 anti-rabbit IgG and Alexa Fluor 568 anti-mouse IgG from Invitrogen; A-11008 and A-11004). After staining, slides were mounted in Aqua Poly/Mount (Polysciences Inc.) and imaged using Zeiss Axioplan 2IE microscope.

Statistics. Data are expressed as mean \pm SEM. T tests were used to analyze data between 2 groups. ANOVA with Bonferroni post-hoc test was used when more than 2 groups were present. All experiments were repeated at least 3 times, and representative experiments are shown. Statistical significance was be achieved when $P<0.05$. Multivariable Cox regression model was used to assess the association between gene expression and time to composite endpoint.

Study approval. All animal protocols were approved by the IACUC at Icahn School of Medicine at Mount Sinai. Archival human biopsy specimens of healthy donor nephrectomies and diabetic nephropathy were collected at Jinglin Hospital, Nanjing University Medical School (Nanjin, China) under a protocol approved by the IRB.

\section{Author contributions}

$\mathrm{JCH}$ and $\mathrm{Z}$. Liu conceived and designed the research experiments. FZ, ZC, LZ, YX, and Z. Li conducted the cell and animal experiments. VN, WJ, MK, and RGN acquired and provided the human data. FZ, ZC, LZ, YX, VN, WJ, MK, RGN, HC, YW, AZ, KL, Z. Liu, and JCH analyzed and interpreted the data. ZC, WJ, RGN, KL, and JCH wrote and edited the manuscript.

\section{Acknowledgments}

ZHC is supported by National Natural Science Foundation of China (no. 81470943) and Jiangsu Basic Research Program (no. BK20181237). RGN is supported by the Intramural Research Program of the NIDDK. KL is supported by NIH R01DK117913. ZHL is supported by National Key Research and Development Program of China (2016YFC0904103), the Major International (Regional) Joint Research Project (no. 81320108007). JCH is supported by VA Merit Award IBX000345C, NIH 1R01DK078897, NIH 1R01DK088541, and NIH P01DK56492. NEPTUNE, U54-DK-083912, is a part of the NIH Rare Disease Clinical Research Network (RDCRN), supported through the collaboration between the Office of Rare Diseases Research (ORDR), National Center for Advancing Translational Sciences (NCATS), and the NIDDK. Additional funding and/or programmatic support for this project have also been provided by the University of Michigan, the NephCure Kidney International, the Halpin Foundation, and NIDDK (P30 DK081943).

Address correspondence to: John Cijiang He, Division of Nephrology, Box 1243, Icahn School of Medicine at Mount Sinai, One Gustave L. Levy Place, New York, New York 10029, USA. Phone: 212.659.1703; Email: cijiang.he@mssm.edu. Or to: Zhihong Liu, National Clinical Research Center of Kidney Diseases, Jinling Hospital, Nanjing University School of Medicine, 305 East Zhongshan Road, Nanjing 210016, China. Phone: 86.25.84801992; Email: liuzhihong@nju.edu.cn.

1. Shankland SJ. The podocyte's response to injury: role in proteinuria and glomerulosclerosis. Kidney Int. 2006;69(12):2131-2147.

2. Greka A, Mundel P. Cell biology and pathology of podocytes. Annu Rev Physiol. 2012;74:299-323.

3. Kikuchi M, Wickman L, Hodgin JB, Wiggins RC. Podometrics as a Potential Clinical Tool for Glomerular Disease Management. Semin Nephrol. 2015;35(3):245-255.

4. Mundel P. Podocyte-Targeted Treatment for Proteinuric Kidney Disease. Semin Nephrol. 2016;36(6):459-462.

5. Wolf G, Chen S, Ziyadeh FN. From the periphery of the glomerular capillary wall toward the center of disease: podocyte injury comes of age in diabetic nephropathy. Diabetes. 2005;54(6):1626-1634.

6. Kriz W, Gretz N, Lemley KV. Progression of glomerular diseases: is the podocyte the culprit? Kidney Int. 1998;54(3):687-697.

7. Zhong F, et al. Protein S Protects against Podocyte Injury in Diabetic Nephropathy. J Am Soc Nephrol. 2018;29(5):1397-1410.

8. Lee IJ, et al. Growth arrest-specific gene 6 (Gas6) levels are elevated in patients with chronic renal failure. Nephrol Dial Transplant. 2012;27(11):4166-4172.

9. van der Meer JH, van der Poll T, van 't Veer C. TAM receptors, Gas6, and protein S: roles in inflammation and hemostasis. Blood. 2014;123(16):2460-2469.

10. Hafizi S, Dahlbäck B. Gas6 and protein S. Vitamin K-dependent ligands for the Axl receptor tyrosine kinase subfamily. FEBS J. 2006;273(23):5231-5244. 
11. Tsou WI, et al. Receptor tyrosine kinases, TYRO3, AXL, and MER, demonstrate distinct patterns and complex regulation of ligand-induced activation. J Biol Chem. 2014;289(37):25750-25763.

12. Studer RA, Opperdoes FR, Nicolaes GA, Mulder AB, Mulder R. Understanding the functional difference between growth arrest-specific protein 6 and protein S: an evolutionary approach. Open Biol. 2014;4(10):140121.

13. Lew ED, et al. Differential TAM receptor-ligand-phospholipid interactions delimit differential TAM bioactivities. Elife $2014 ; 3$ e03385.

14. Nagai K, et al. Growth arrest-specific gene 6 is involved in glomerular hypertrophy in the early stage of diabetic nephropathy. J Biol Chem. 2003;278(20):18229-18234.

15. Nagai K, et al. Gas6 induces Akt/mTOR-mediated mesangial hypertrophy in diabetic nephropathy. Kidney Int. 2005;68(2):552-561.

16. Burstyn-Cohen T, Heeb MJ, Lemke G. Lack of protein S in mice causes embryonic lethal coagulopathy and vascular dysgenesis J Clin Invest. 2009;119(10):2942-2953.

17. Saller F, et al. Generation and phenotypic analysis of protein S-deficient mice. Blood. 2009;114(11):2307-2314.

18. Fraineau S, et al. The vitamin K-dependent anticoagulant factor, protein S, inhibits multiple VEGF-A-induced angiogenesis events in a Mer- and SHP2-dependent manner. Blood. 2012;120(25):5073-5083.

19. Fan Y, et al. Temporal profile of the renal transcriptome of HIV-1 transgenic mice during disease progression. PLoS ONE 2014;9(3):e93019.

20. Gadegbeku CA, et al. Design of the Nephrotic Syndrome Study Network (NEPTUNE) to evaluate primary glomerular nephropathy by a multidisciplinary approach. Kidney Int. 2013;83(4):749-756.

21. Kramer-Zucker AG, Wiessner S, Jensen AM, Drummond IA. Organization of the pronephric filtration apparatus in zebrafish requires Nephrin, Podocin and the FERM domain protein Mosaic eyes. Dev Biol. 2005;285(2):316-329.

22. Li X, Dai Y, Chuang PY, He JC. Induction of retinol dehydrogenase 9 expression in podocytes attenuates kidney injury. J Am Soc Nephrol. 2014;25(9):1933-1941.

23. Hong Q, et al. Increased podocyte Sirtuin-1 function attenuates diabetic kidney injury. Kidney Int. 2018;93(6):1330-1343.

24. Zheng S, et al. Development of late-stage diabetic nephropathy in OVE26 diabetic mice. Diabetes. 2004;53(12):3248-3257.

25. Xu J, Huang Y, Li F, Zheng S, Epstein PN. FVB mouse genotype confers susceptibility to OVE26 diabetic albuminuria. Am J Physiol Renal Physiol. 2010;299(3):F487-F494.

26. Berthier CC, et al. Enhanced expression of Janus kinase-signal transducer and activator of transcription pathway members in human diabetic nephropathy. Diabetes. 2009;58(2):469-477.

27. Schmid H, et al. Modular activation of nuclear factor-kappaB transcriptional programs in human diabetic nephropathy. Diabetes 2006;55(11):2993-3003.

28. Liu R, et al. Role of transcription factor acetylation in diabetic kidney disease. Diabetes. 2014;63(7):2440-2453.

29. Medapalli RK, He JC, Klotman PE. HIV-associated nephropathy: pathogenesis. Curr Opin Nephrol Hypertens. 2011;20(3):306-311.

30. Lemke G. Biology of the TAM receptors. Cold Spring Harb Perspect Biol. 2013;5(11):a009076.

31. Chuang PY, He JC. Signaling in regulation of podocyte phenotypes. Nephron Physiol. 2009;111(2):p9-15.

32. Steffes MW, Schmidt D, McCrery R, Basgen JM, International Diabetic Nephropathy Study Group. Glomerular cell number in normal subjects and in type 1 diabetic patients. Kidney Int. 2001;59(6):2104-2113.

33. Meyer TW, Bennett PH, Nelson RG. Podocyte number predicts long-term urinary albumin excretion in Pima Indians with Type II diabetes and microalbuminuria. Diabetologia. 1999;42(11):1341-1344.

34. Macconi D, et al. Pathophysiologic implications of reduced podocyte number in a rat model of progressive glomerular injury. Am J Pathol. 2006;168(1):42-54.

35. Pagtalunan ME, et al. Podocyte loss and progressive glomerular injury in type II diabetes. J Clin Invest. 1997;99(2):342-348.

36. Faul C, et al. The actin cytoskeleton of kidney podocytes is a direct target of the antiproteinuric effect of cyclosporine A. Nat Med. 2008;14(9):931-938.

37. Guess A, Agrawal S, Wei CC, Ransom RF, Benndorf R, Smoyer WE. Dose- and time-dependent glucocorticoid receptor signaling in podocytes. Am J Physiol Renal Physiol. 2010;299(4):F845-F853.

38. Fornoni A. Proteinuria, the podocyte, and insulin resistance. N Engl J Med. 2010;363(21):2068-2069.

39. Mallipattu SK, He JC. The beneficial role of retinoids in glomerular disease. Front Med (Lausanne). 2015;2:16.

40. Garsen M, et al. Vitamin D attenuates proteinuria by inhibition of heparanase expression in the podocyte. J Pathol. 2015;237(4):472-481.

41. Nelson RG, Meyer TW, Myers BD, Bennett PH. Clinical and pathological course of renal disease in non-insulin-dependent diabetes mellitus: the Pima Indian experience. Semin Nephrol. 1997;17(2):124-131.

42. Woroniecka KI, Park AS, Mohtat D, Thomas DB, Pullman JM, Susztak K. Transcriptome analysis of human diabetic kidney disease. Diabetes. 2011;60(9):2354-2369.

43. Hodgin JB, et al. Identification of cross-species shared transcriptional networks of diabetic nephropathy in human and mouse glomeruli. Diabetes. 2013;62(1):299-308.

44. Wiggins JE, et al. NFkappaB promotes inflammation, coagulation, and fibrosis in the aging glomerulus. J Am Soc Nephrol. 2010;21(4):587-597.

45. Bridgewater DJ, Ho J, Sauro V, Matsell DG. Insulin-like growth factors inhibit podocyte apoptosis through the PI3 kinase pathway. Kidney Int. 2005;67(4):1308-1314

46. Tejada T, et al. Failure to phosphorylate AKT in podocytes from mice with early diabetic nephropathy promotes cell death. Kidney Int. 2008;73(12):1385-1393.

47. Ju W, et al. Tissue transcriptome-driven identification of epidermal growth factor as a chronic kidney disease biomarker. $S_{c i}$ Transl Med. 2015;7(316):316ra193.

48. Mariani LH, et al. Interstitial fibrosis scored on whole-slide digital imaging of kidney biopsies is a predictor of outcome in proteinuric glomerulopathies. Nephrol Dial Transplant. 2018;33(2):310-318.

49. Potapova TA, Sivakumar S, Flynn JN, Li R, Gorbsky GJ. Mitotic progression becomes irreversible in prometaphase and collapses when Wee1 and Cdc25 are inhibited. Mol Biol Cell. 2011;22(8):1191-1206. 
50. Mallipattu SK, et al. Kruppel-like factor 15 (KLF15) is a key regulator of podocyte differentiation. J Biol Chem. 2012;287(23):19122-19135.

51. Ratnam KK, et al. Role of the retinoic acid receptor- $\alpha$ in HIV-associated nephropathy. Kidney Int. 2011;79(6):624-634.

52. Koop K, et al. Expression of podocyte-associated molecules in acquired human kidney diseases. J Am Soc Nephrol. 2003;14(8):2063-2071.

53. Takemoto M, et al. A new method for large scale isolation of kidney glomeruli from mice. Am J Pathol. 2002;161(3):799-805.

54. Saleem MA, et al. A conditionally immortalized human podocyte cell line demonstrating nephrin and podocin expression. J Am Soc Nephrol. 2002;13(3):630-638.

55. Gassmann M, Grenacher B, Rohde B, Vogel J. Quantifying Western blots: pitfalls of densitometry. Electrophoresis. 2009;30(11):1845-1855 